\title{
Infinite interval exchange transformations from shifts
}

\author{
LUIS-MIGUEL LOPEZ $\dagger$ and PHILIPPE NARBEL $\ddagger$ \\ $\dagger$ Tokyo University of Social Welfare, Isesaki, 372-0831 Gunma, Japan \\ (e-mail:lopez@ed.tokyo-fukushi.ac.jp) \\ $\ddagger$ LaBRI, University of Bordeaux, 33405 Talence, France \\ (e-mail: narbel@labri.fr)
}

(Received 20 June 2015 and accepted in revised form 19 October 2015)

\begin{abstract}
We show that minimal shifts with zero topological entropy are topologically conjugate to interval exchange transformations, which are generally infinite. When these shifts have linear factor complexity (linear block growth), the conjugate interval exchanges are proved to satisfy strong finiteness properties.
\end{abstract}

\section{Introduction}

Interval exchange transformations (IETs) are maps over $I=[0,1)$ which can be defined as permutations of intervals partitioning $I$. In the finite case, these maps are just piecewise isometries of $I$ onto itself, or equivalently, injective measure-preserving maps having only a finite number of discontinuities. They happen to be a fundamental notion in dynamical systems and ergodic theory [CFS82, Mañ87, HK02]. Another important notion in the same context is the shift $\sigma$ on the set $A^{\mathbb{N}}$ of infinite words (symbolic sequences) over a finite alphabet $A$. This simple continuous map consists of erasing the first letter of its argument. The pair $\left(A^{\mathbb{N}}, \sigma\right)$ forms a basic topological dynamical system, where $A^{\mathbb{N}}$ is then called the full shift over $A$. If $L$ is a closed $\sigma$-invariant subset of $A^{\mathbb{N}}$, the pair $(L, \sigma)$ also induces such a system, where $L$ is then just called a shift [LM95, Kit98]. The topological entropy of a shift $L$ depends on the factor complexity (block growth) of $L$ [MH38, Par66, CN10], i.e. the map $p_{L}$ on $\mathbb{N}^{*}$ giving for each $n$ the number of distinct length- $n$ factors (subblocks) occurring in the words of $L$. Now, a known general relationship between all the above concepts is the following: the support $I$ of a finite IET can be embedded as a subset into a measured compact space in such a way that the IET extends to a measurepreserving continuous map, whose natural symbolic conjugate is a shift $L$ with a factor complexity bounded by an affine function (thus $L$ has zero topological entropy) [Kea75]. The main idea of this paper is to study this relationship the other way around, that is, 
starting from shifts to build topologically conjugate IETs, generally with infinitely many discontinuities, and using factor complexity to determine families of these IETs.

Thus, given a shift $L$, we first describe an ordered measured compact space $X_{L}$ containing $I$ and a continuous self-map $f_{L}$ on $X_{L}$ in such a way that $(L, \sigma)$ is topologically conjugate to $\left(X_{L}, f_{L}\right)$. Next, we show how $f_{L}$ can be seen as an extension over $X_{L}$ of an IET $T_{L}$ over $I$. We shall mostly consider aperiodic minimal shifts, i.e. shifts with no periodic word and containing no proper shift. With this respect, the first main result we prove is the following.

THEOREM 1. Let L be a measured minimal aperiodic shift with zero topological entropy. Then $(L, \sigma)$ is topologically conjugate to $\left(X_{L}, f_{L}\right)$, where $f_{L}$ is the extension over $X_{L}$ of an IET $T_{L}$ on $I \subset X_{L}$.

This result is to be put into perspective with the fact that aperiodic measurepreserving transformations of a Lebesgue space are known to be isomorphic to infinite IETs [AOW85]. Besides the fact it defines a tight relationship between shifts and transformations over $[0,1)$, Theorem 1 is about topological isomorphismsi.e. homeomorphisms - and not only about isomorphisms - i.e. measure-preserving maps. Another comparison point is that the conjugate IETs given by Theorem 1 can have up to a null measure infinite set of discontinuities, while being always piecewise increasing. Also, the construction behind these conjugate maps has the following consistency property: starting with a piecewise increasing IET $T$, and coding its dynamics after a finite monotonicity partition of $I$, a shift is obtained to which Theorem 1 applies, yielding an IET coinciding with $T$ (see Proposition 3.3.3 further).

The paper focuses next on aperiodic minimal shifts $L$ with linear complexity [Fog02, CN10], i.e. $p_{L}(n)=O(n)$, forming a family of shifts with zero topological entropy, which contains all the simplest non-trivial ones. In particular, these linear complexity shifts include not only the conjugate shifts obtained from the natural coding of finite IETs, but also the shifts associated with primitive substitutions [Qué10]. Now, the simplicity behind linear complexity is reflected in the conjugate IETs. In this case, a conjugate IET given by Theorem 1 has three properties: in addition to being piecewise increasing, its discontinuities may accumulate only at a finite set, and all these discontinuities belong to only finitely many distinct iterates (full orbits) of the IET. We call an IET satisfying these properties almost finite. The second main result we prove is the following variation of Theorem 1.

THEOREM 2. Let $L$ be a measured minimal aperiodic shift with linear complexity. Then $(L, \sigma)$ is topologically conjugate to $\left(X_{L}, f_{L}\right)$, where $f_{L}$ is the extension over $X_{L}$ of an almost finite IET $T_{L}$ on $I \subset X_{L}$.

The above theorems can be constructive. In particular, we describe a technique to exhibit $\sigma$-invariant measures on the shifts $L$ for which the conjugate IETs can be explicitly built (see Proposition 5.1.3, and the examples illustrating it).

\section{Basic definitions}

\subsection{Interval exchange transformations.}


Definition 1. An orientation-preserving finite interval exchange transformation $\dagger$ (IET) is a map $T: I \rightarrow I$, where $I=[0,1)$, with:

- a finite set $\left\{x_{i}\right\}_{i=0, \ldots, m-1}$ of discontinuities, denoted by $\mathcal{D}$, with $x_{0}=0<x_{1}<\cdots<$ $x_{m-1}<x_{m}=1$, which determines an ordered partition $\bigsqcup_{i=0}^{m-1} I_{i}$ of $I$ formed by the right-open intervals $I_{i}=\left[x_{i}, x_{i+1}\right)$,

such that:

- $T$ is injective, and $T$ is a translation on each interval $I_{i}$, i.e. for each $i=0, \ldots, m-1$, there exists $k_{i} \in \mathbb{R}$ such that for all $x \in I_{i}, T(x)=x+k_{i}$.

Such a finite IET $T$ is just a piecewise order-preserving isometry of $I$. It is rightcontinuous, and measure-preserving, being injective and with derivative 1 on $I \backslash \mathcal{D}$. A usual way of interpreting $T$ - giving its name to the notion -is the following: the intervals $I_{i}$, as ordered components of the partition $\bigsqcup_{i=0}^{m-1} I_{i}$ of $I$, are permuted in the image of $T$ so as to form another partition $\bigsqcup_{i=0}^{m-1} I_{\pi(i)}$ of $I$, where $\pi$ is the permutation over $\{0, \ldots, m-1\}$ induced by the $k_{i}$ determining $T$. Indeed an IET can also be defined by a pair $(\lambda, \pi)$, where $\lambda$ is the vector of the lengths of the $I_{i}$, and $\pi$ is a permutation of the $I_{i}$. Note that, given an IET, the partition of $I$ can be refined by using any finite set $B \supset \mathcal{D}$ of points, and such a refinement can also be used in Definition 1 by replacing $\mathcal{D}$ with $B$. The points determining a specific partition of an IET $T$ are then usually called the separation points of $T$.

The above definition of an IET can be extended to include infinity as follows.

Definition 2. An orientation-preserving infinite interval exchange transformation is a map $T: I \rightarrow I$, where $I=[0,1)$, with:

- an infinite set $Y$ of discontinuities such that the closure of $Y$ in $I$, denoted by $\mathcal{D}$, has null measure and determines an ordered partition of $I$ formed by:

(i) the right-open intervals $\left[x, x^{\prime}\right)$ with $x \in \mathcal{D}, x^{\prime} \in \mathcal{D} \cup\{1\},\left[x, x^{\prime}\right) \cap \mathcal{D}=\{x\}$;

(ii) the set $\mathcal{D}_{\text {acc, } r}$ of the accumulation points of $\mathcal{D}$ from the right, such that:

- $T$ is injective on $I \backslash \mathcal{D}_{\text {acc, } r}$, right-continuous on $I$, and $T$ is a translation on each rightopen interval of the above partition $\ddagger$.

An infinite IET $T$ is also measure-preserving, being injective with derivative 1 on $I \backslash \mathcal{D}$, and $\mathcal{D}$ being of null measure. Since $T$ is right-continuous on $I$, its values on $\mathcal{D}_{\text {acc, } r}$ are determined by its values on $I \backslash \mathcal{D}_{\text {acc, } r}$. Like in the finite case, $\mathcal{D}$ can be replaced in the above definition by any null-measure set $B$ of separation points such that $B \supset \mathcal{D}$, where $B \cup\{1\}$ is compact. Note that we can use a null-measure infinite set $B$ for finite IETs too.

2.2. Words. Let $A$ be a finite alphabet, let $A^{+}$be the set of finite words over $A$, let $A^{*}$ be $A^{+}$with the empty word $\epsilon$, and let $A^{\mathbb{N}}$ be the set of infinite words over $A$. The alphabet $A$ can be endowed with the discrete topology, and the resulting product topology on $A^{\mathbb{N}}$ is metrizable and compact. The usual associated metric is the Cantor

$\dagger$ In this paper, it is sufficient to consider the orientation-preserving case only, i.e. interval exchange transformations with $(+1)$ slopes and no $(-1)$ slopes as in the general case [Mañ87].

\$ Infinite IETs are considered in different ways in the literature depending on the authors, e.g. in [AOW85], the discontinuities accumulate only at 1, whereas in [Hoo15], the discontinuities are countable and the partitioned interval may have infinite length. 
metric: if $w=a_{0} a_{1} a_{2} \ldots$ and $w^{\prime}=a_{0}^{\prime} a_{1}^{\prime} a_{2}^{\prime} \ldots$ in $A^{\mathbb{N}}$, with $a_{i}, a_{i}^{\prime} \in A$, their distance is 0 if they are equal and $2^{-k}$ if not, where $k$ is the smallest non-negative integer for which $a_{k} \neq a_{k}^{\prime}$. Let $v=v_{0} v_{1} \ldots v_{n} \in A^{*}$, with $v_{i} \in A$, then its cylinder set (for short, cylinder) is $\operatorname{Cyl}(v)=\left\{w=a_{0} a_{1} a_{2} \ldots \in A^{\mathbb{N}} \mid a_{0}=v_{0}, a_{1}=v_{1}, \ldots, a_{n}=v_{n}\right\}$. A cylinder is a clopen set, and the collection of all the cylinders forms a basis for the topology on $A^{\mathbb{N}}$. For $L \subseteq A^{\mathbb{N}}$ and $v \in A^{*}$, the cylinder in the subspace topology is $\operatorname{Cyl}_{L}(v)=\operatorname{Cyl}(v) \cap L$. Let $\leq$ be an order over $A$, propagated to all the words in $A^{+}$and $A^{\mathbb{N}}$ as the lexicographic order. Then for every $w, w^{\prime} \in L$ with $w \leq w^{\prime}$, the word interval $\left[w, w^{\prime}\right]$ in $L$ is $\left\{w^{\prime \prime} \in L \mid w \leq w^{\prime \prime} \leq w^{\prime}\right\}$. Note that the induced order topology is the same as the product topology.

A factor (or subblock) of a word $w$ is a finite word $v$ such that $w=w^{\prime} v w^{\prime \prime}$, where $w^{\prime}, w^{\prime \prime}$ are possibly empty, and where $w^{\prime} v w^{\prime \prime}$ denotes the word concatenation of $w^{\prime}, v$ and $w^{\prime \prime}$. The length of $v$ is denoted by $|v|$. The set of all distinct factors of a word $w$ is denoted by Fact ${ }_{w}$, and for a set $L \subseteq A^{\mathbb{N}}$, by Fact b $_{L}=\bigcup_{w \in L}$ Fact $_{w}$. For each $n \in \mathbb{N}^{*}$, the set of all the distinct factors $v$ of $w$ such that $|v|=n$ is denoted by $\operatorname{Fact}_{w}(n)$, and for $L \subseteq A^{\mathbb{N}}$, by $\operatorname{Fact}_{L}(n)=\bigcup_{w \in L} \operatorname{Fact}_{w}(n)$. The (factor) complexity (or block growth) [MH38, CN10] of a word $w$ is the function $p_{w}: \mathbb{N}^{*} \rightarrow \mathbb{N}^{*}$, with $p_{w}(n)=\left|\operatorname{Fact}_{w}(n)\right|$, where $|\cdot|$ for a set denotes its cardinality, and for $L \subseteq A^{\mathbb{N}}$, it is $p_{L}(n)=\left|\operatorname{Fact}_{L}(n)\right|$. When $L$ is a shift, its topological entropy [Par66] is defined as $\lim _{n \rightarrow \infty} \log \left(p_{L}(n)\right) / n$ (which exists, since $\log \left(p_{L}(n)\right)$ is subadditive).

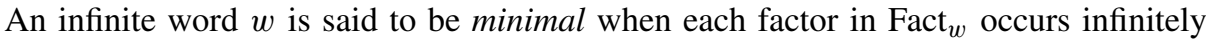
often in $w$ with bounded gaps, i.e. for each factor $v$ of $w=a_{0} a_{1}, \ldots$, the ordered sequence of distinct indexes $\left\{n_{j}\right\}_{j \in \mathbb{N}}$ such that $a_{n_{j}} \ldots a_{n_{j}+|v|-1}=v$ is infinite, and $n_{j+1}-n_{j}$ is bounded independently of $j$ [MH38]. Such a word $w$ is minimal aperiodic when it is not periodic, i.e. when there is no factor $v$ such that $w=v^{\omega}$. A set of finite words is prolongable if all its words can always be concatenated with letters to the right and to the left so that the resulting words still belong to the set. When $L \subseteq A^{\mathbb{N}}$ is made of minimal words, Fact $_{L}$ is prolongable.

2.3. Dynamical systems and shifts. Considering a self-map $f: X \rightarrow X$, where $X$ is a measured space and where $f$ is measurable, the pair $(X, f)$ is called a measured dynamical system. When $X$ is a topological space and $f$ is continuous, $(X, f)$ is called a topological dynamical system. The shift map $\sigma$ over $A^{\mathbb{N}}$ sends $a_{0} a_{1} \ldots$ to $a_{0}^{\prime} a_{1}^{\prime}, \ldots$, where $a_{i}^{\prime}=a_{i+1}$ for every $i \in \mathbb{N}$, and it is a continuous map over $A^{\mathbb{N}}$. A shift space (or simply a shift) [LM95] is a closed $\sigma$-invariant set of words $L$ in $A^{\mathbb{N}}$, and accordingly, $(L, \sigma)$ is an instance of a topological dynamical system. For a dynamical system $(X, f)$, the (positive) orbit of a point $x \in X$ is $\left\{f^{n}(x)\right\}_{n \in \mathbb{N}}$. When $f$ is invertible, the full orbit is $\left\{f^{n}(x)\right\}_{n \in \mathbb{Z}}$, and when $f$ is not invertible, it is the $\sim$-class of points in $X$ containing $x$, where $x^{\prime} \sim x$ if there are $n_{1}, n_{2} \geq 0$ such that $f^{n_{1}}\left(x^{\prime}\right)=f^{n_{2}}(x)$ [KST01]. A continuous map $f: X \rightarrow X$ is said to be minimal if there is no non-empty closed proper subset $X^{\prime} \subsetneq X$ such that $f\left(X^{\prime}\right)=X^{\prime}$, or equivalently if the orbit of each $x \in X$ is dense in $X$. The map $f$ is minimal aperiodic if $X$ does not consist of a single periodic orbit. Accordingly, a minimal shift is a shift containing no proper shift, and a minimal aperiodic shift is a shift which is not made of the orbit of one single periodic word. If a word $w$ belongs to a minimal shift $L$, it is minimal as a word, and Fact $_{w}=$ Fact $_{L}$. 
For two measured dynamical systems $(X, f)$ and $(Y, g)$, if there exists a measurable isomorphism $\phi: X \rightarrow Y$ such that $\phi \circ f=g \circ \phi$, then $(X, f)$ and $(Y, g)$ are said to be conjugate by $\phi$. When $(X, f)$ and $(Y, g)$ are topological dynamical systems and $\phi$ is a continuous onto map (respectively a homeomorphism), $(X, f)$ and $(Y, g)$ are said to be topologically semi-conjugate (respectively topologically conjugate) by $\phi$.

\section{From shifts to interval exchange transformations}

3.1. The conjugacies. We start here by presenting basic results about how to embed ordered measured spaces coming from shifts into $[0,1]$.

For any dynamical system $(X, f)$, where $X$ is a compact space and $f$ is a continuous self-map on $X$, there exist Borel probability measures on $X$ which are $f$-invariant [HK02]. If $f$ is minimal aperiodic, any such measure is non-atomic and takes positive values on open sets. Borel measures on compact sets behave well with respect to approximating measurable sets by open and/or closed sets since they are regular, i.e. for any measurable set $E, \mu(E)=\sup \left\{\mu\left(E^{\prime}\right) \mid E^{\prime}\right.$ compact, $\left.E^{\prime} \subseteq E\right\}$ and $\mu(E)=\inf \left\{\mu\left(E^{\prime}\right) \mid E^{\prime}\right.$ open, $E \subseteq$ $\left.E^{\prime}\right\}$. For a regular measure, to be non-atomic is equivalent to every singleton having measure 0 .

From now on, $L$ denotes a shift lexicographically ordered by $\leq$, and endowed with a $\sigma$-invariant Borel probability measure $\mu$ which is non-atomic (so that the measure of any single word in $L$ is zero), positive on cylinders, and regular. If $L$ is a minimal aperiodic shift, as just said above, such measures exist.

Remark 3.1.1. There is no isolated word in $L$.

Proof. If there was an isolated word $w$ in $L$, there would exist a prefix $v$ of $w$ such that $\mathrm{Cyl}_{L}(v)$ contains only $w$, but then $\{w\}$ would have positive measure.

Using $\mu$ and the order $\leq$ over $L$, and denoting the smallest word of $L$ by $w_{L \text {, min }}$, we define:

$$
\begin{aligned}
\phi_{\mu}: \quad L & \rightarrow \bar{I}=[0,1] \\
w & \mapsto \mu\left(\left[w_{L, \min }, w\right]\right) .
\end{aligned}
$$

\section{LEMMA 3.1.2. $\phi_{\mu}$ is a monotonic non-decreasing continuous map.}

Proof. Since $\mu$ is a measure which takes its values in $[0,1], \phi_{\mu}$ is monotonic nondecreasing. For continuity, the involved spaces being compact, we just check that the image by $\phi_{\mu}$ of every sequence in $L$ converging to some $w \in L$ is a sequence converging to $\phi_{\mu}(w)$. Let $\mathcal{S}$ be such a sequence, from which we extract two subsequences $\left\{w_{i}^{+}\right\}$ and $\left\{w_{i}^{-}\right\}$, formed by decreasing words greater than $w$ and by increasing words smaller than $w$, respectively. At least one of them is infinite, say $\left\{w_{i}^{+}\right\}$, and $\left[w_{L, \min }, w\right]=$ $\bigcap_{i}\left[w_{L, \min }, w_{i}^{+}\right)$. Since $\mu$ is regular, $\mu\left(\left[w_{L, \min }, w\right]\right)=\phi_{\mu}(w)=\inf _{i} \mu\left(\left[w_{L, \min }, w_{i}^{+}\right)\right)$, and since $\mu$ is zero on single words for all $i>0, \mu\left(\left[w_{L, \min }, w_{i}^{+}\right]\right)=\phi_{\mu}\left(w_{i}^{+}\right)$, thus $\inf _{i} \phi_{\mu}\left(w_{i}^{+}\right)=\phi_{\mu}(w)$. Hence since $\phi_{\mu}$ is non-decreasing, $\left\{\phi_{\mu}\left(w_{i}^{+}\right)\right\}$converges to $\phi_{\mu}(w)$, whatever the choice of $\left\{w_{i}^{+}\right\}$. If $\left\{w_{i}^{-}\right\}$is also infinite, similar arguments as for $\left\{w_{i}^{+}\right\}$apply using suprema instead of infima, whence the result. 
LEMMA 3.1.3. $\phi_{\mu}$ is a surjective map.

Proof. Since $L$ is compact and $\phi_{\mu}$ is continuous, $\bar{I} \backslash \phi_{\mu}(L)$ is open, and if not empty it is a disjoint union of open intervals, since 0 and 1 belong to $\phi_{\mu}(L)$. Let $J=\left(x, x^{\prime}\right)$, with $x, x^{\prime} \in \phi_{\mu}(L)$, be one of these intervals, and let $w_{x, \sup }=\sup \left\{w \in L \mid \phi_{\mu}(w)=x\right\}$ and $w_{x^{\prime}, \text { inf }}=\inf \left\{w \in L \mid \phi_{\mu}(w)=x^{\prime}\right\}$. Since $x<x^{\prime}$ and since $\phi_{\mu}$ is non-decreasing, $w_{x, \text { sup }}<$

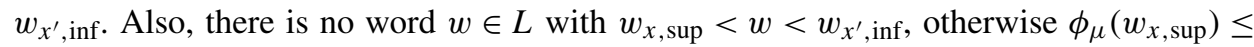
$\phi_{\mu}(w) \leq \phi_{\mu}\left(w_{x^{\prime}, \text { inf }}\right)$, and by the definition of $J$ we would have $\phi_{\mu}(w)=x$ or $\phi_{\mu}(w)=$ $x^{\prime}$, contradicting the definitions of $w_{x, \text { sup }}$ or $w_{x^{\prime}, \text { inf }}$. Now, $\phi_{\mu}\left(w_{x^{\prime}, \text { inf }}\right)-\phi_{\mu}\left(w_{x, \text { sup }}\right)=$ $\mu\left(\left[w_{L, \min }, w_{x^{\prime}, \text { inf }}\right]\right)-\mu\left(\left[w_{L, \min }, w_{x, \text { sup }}\right]\right)=\mu\left(\left(w_{x, \text { sup }}, w_{x^{\prime}, \text { inf }}\right]\right)$, which is equal to the non-zero length of $J$. But we just have checked that $\left(w_{x, \text { sup }}, w_{x^{\prime}, \text { inf }}\right]=\left\{w_{x^{\prime}, \text { inf }}\right\}$ and $\mu$ is zero on single words. Hence there is no interval such as $J$, so $\phi_{\mu}$ is onto.

We now characterize the points where $\phi_{\mu}$ is non-injective. For each $n>0$, the length- $n$ factor set $\operatorname{Fact}_{L}(n)$ induces an ordered finite partition of $L$ defined as

$$
\mathrm{CYL}_{L}(n): \quad L=\bigsqcup_{v \in \operatorname{Fact}_{L}(n)} \operatorname{Cyl}_{L}(v),
$$

where the $\operatorname{Cyl}_{L}(v)$ are ordered in $L$ according to the lexicographic order of the $v$ in $\operatorname{Fact}_{L}(n)$. By compactness, each $\mathrm{Cyl}_{L}(v)$ has two endpoints: its smallest and its greatest words. We say that $w, w^{\prime} \in L$ (respectively $\left.\operatorname{Cyl}(u), \operatorname{Cyl}\left(u^{\prime}\right) \in \operatorname{CYL}_{L}(n), n>0\right)$ are consecutive if $w<w^{\prime}$ (respectively $\operatorname{Cyl}(u)<\operatorname{Cyl}\left(u^{\prime}\right)$ ) and if there is no word $w^{\prime \prime} \in L$ such that $w<w^{\prime \prime}<w^{\prime}$ (respectively $\operatorname{Cyl}(u)<w^{\prime \prime}<\operatorname{Cyl}\left(u^{\prime}\right)$ ).

Lemma 3.1.4. Let $w, w^{\prime} \in L$. Then $w, w^{\prime}$ are consecutive if and only if for some $n>0$ they are endpoints, respectively the greatest and the smallest words, of two consecutive cylinders of $\mathrm{CYL}_{L}(n)$.

Proof. $(\Leftarrow)$ Trivial. $(\Rightarrow)$ Assume $w<w^{\prime}$, with $w, w^{\prime}$ consecutive. Let $u \in A^{*}$ be their longest common prefix, so that $w=u a_{1} a_{2} \ldots$ and $w^{\prime}=u a_{1}^{\prime} a_{2}^{\prime}, \ldots$, with $a_{i}, a_{i}^{\prime} \in A$. Thus $w, w^{\prime} \in \operatorname{Cyl}_{L}(u)$ and $a_{1}<a_{1}^{\prime}$. Since there is no word between $w$ and $w^{\prime}$, the prefix $u a_{1} \ldots a_{j-1} a_{j}$ of $w$ for each $j>1$ is also the greatest prefix for all the words in $\mathrm{Cyl}_{L}\left(u a_{1}\right)$. Otherwise, there would be a factor $u a_{1} v \in \operatorname{Fact}_{L}(|u|+j), v \in A^{+}$, such that $u a_{1} \ldots a_{j-1} a_{j}<u a_{1} v$ as a prefix of a word $w^{\prime \prime}=u a_{1} v \ldots \in L$. But then $w<w^{\prime \prime}<w^{\prime}$, which is impossible. Thus $w$ is the greatest word in $\operatorname{Cyl}_{L}\left(u a_{1}\right)$. By similar arguments, $w^{\prime}$ is the smallest word in $\operatorname{Cyl}_{L}\left(u a_{1}^{\prime}\right)$. Finally, there is no other $a \in A$ with $a_{1}<a<a_{1}^{\prime}$, such that there is $w^{\prime \prime \prime}=u a \ldots \in L$, otherwise again $w<w^{\prime \prime \prime}<w^{\prime}$. Hence $\operatorname{Cyl}_{L}\left(u a_{1}\right)$ and $\mathrm{Cyl}_{L}\left(u a_{1}^{\prime}\right)$ are consecutive cylinders in $\mathrm{CYL}_{L}(|u|+1)$.

Lemma 3.1.5. Let $w, w^{\prime} \in L$. Then $\phi_{\mu}$ is non-injective on $\left\{w, w^{\prime}\right\}$ if and only if $w, w^{\prime}$ are consecutive.

Proof. $(\Leftarrow)$ Assume $w<w^{\prime}$, with $w, w^{\prime}$ consecutive. Then we have $\phi_{\mu}\left(w^{\prime}\right)-\phi_{\mu}(w)=$ $\mu\left(\left[w_{L, \min }, w^{\prime}\right]\right)-\mu\left(\left[w_{L, \min }, w\right]\right)=\mu\left(\left(w, w^{\prime}\right]\right)=\mu\left(\left\{w^{\prime}\right\}\right)=0$, since $\mu$ is zero on single words. $(\Rightarrow)$ Assume $w<w^{\prime}$, with $w, w^{\prime}$ non-consecutive. Thus there exists $w^{\prime \prime} \in L$ with $w<w^{\prime \prime}<w^{\prime}$, so that there is a length- $n$ prefix $u$ of $w^{\prime \prime}$, with $n>0$, distinct from the length- $n$ prefixes of $w$ and $w^{\prime}$. But then, every $w^{\prime \prime \prime} \in \operatorname{Cyl}_{L}(u)$ is such that $w<w^{\prime \prime \prime}<w^{\prime}$, 
and we have $\phi_{\mu}\left(w^{\prime}\right)-\phi_{\mu}(w)=\mu\left(\left(w, w^{\prime}\right]\right) \geq \mu\left(\mathrm{Cyl}_{L}(u)\right)>0$, since $\mu$ is positive on cylinders.

Thus $\phi_{\mu}^{-1}(x), x \in \bar{I}$, consists of either one or two words in $L$, and a consequence of Lemma 3.1.4 is that the set of points with two-word preimages is countable.

Let us then transform $\phi_{\mu}$ into an injective map by embedding $\bar{I}$ as a subset of a larger compact space (using a similar construction to the classic one for transforming piecewise continuous self-maps into homeomorphisms, as in e.g. [Kea75] for IETs). We first define the following spaces and maps.

- $Z_{0} \subset \bar{I}$ denotes the image by $\phi_{\mu}$ of the set of points where $\phi_{\mu}$ is not injective.

- $Z_{0}^{-}$denotes a copy of $Z_{0}$.

- $X_{L}$ denotes $\bar{I} \sqcup Z_{0}^{-}$, ordered in such a way that each point in $Z_{0}$ lies to the right of its copy in $Z_{0}^{-}$, with no other point in between. We endow $X_{L}$ with the order topology for this order relation.

- $\iota: \bar{I} \rightarrow X_{L}$ denotes the inclusion map. It is increasing and right-continuous on $I$.

- $\kappa: X_{L} \rightarrow \bar{I}$ denotes the canonical map associated with the equivalence relation in $X_{L}$ which identifies each point in $Z_{0}$ with its copy in $Z_{0}^{-}$. It is a non-decreasing continuous map, and it is onto. Accordingly, $\kappa \circ \iota$ is the identity map on $\bar{I}$.

Having in mind Lemma 3.1.5 we then define the following map from $\phi_{\mu}$ :

$$
\begin{aligned}
\phi: L & \rightarrow X_{L} \\
w & \mapsto \begin{cases}\iota\left(\phi_{\mu}(w)\right) & \text { if } \phi_{\mu}(w) \notin Z_{0}, \text { or } \\
& \text { if } \phi_{\mu}(w) \in Z_{0}, \text { with } \phi_{\mu}^{-1}\left(\phi_{\mu}(w)\right)=\left\{w, w^{\prime}\right\}, w>w^{\prime}, \\
\iota\left(\phi_{\mu}(w)\right)^{-} & \text {if } \phi_{\mu}(w) \in Z_{0}, \text { with } \phi_{\mu}^{-1}\left(\phi_{\mu}(w)\right)=\left\{w, w^{\prime}\right\}, w<w^{\prime}, \\
& \text { where } \iota\left(\phi_{\mu}(w)\right)^{-} \text {is the copy of } \iota\left(\phi_{\mu}(w)\right) \text { in } Z_{0}^{-} .\end{cases}
\end{aligned}
$$

Note that $\kappa \circ \phi=\phi_{\mu}$.

LEMMA 3.1.6. $\phi$ is an increasing homeomorphism.

Proof. By Lemma 3.1.2, $\phi$ is increasing where $\phi_{\mu}$ is injective since $\phi=\phi_{\mu}$ on these points. Where $\phi_{\mu}$ is not injective, that is, for each $x \in Z_{0}$ where $\phi_{\mu}^{-1}(x)=\left\{w, w^{\prime}\right\}$ for some $w, w^{\prime} \in L$ with $w<w^{\prime}$, then by definition of $X_{L}$ and $\phi$ we have $\phi(w)<\phi\left(w^{\prime}\right)$. Therefore $\phi$ is everywhere increasing, thus into. Since $X_{L}=\iota(\bar{I}) \sqcup Z_{0}^{-}, \phi$ is also onto. Hence $\phi$ is a monotonic bijection between two totally ordered sets endowed with their respective order topologies, so it is a homeomorphism.

We define two more maps:

$$
\begin{aligned}
& f_{L}: X_{L} \rightarrow X_{L} \quad T_{L}: \bar{I} \rightarrow \bar{I} \\
& x \mapsto \phi\left(\sigma\left(\phi^{-1}(x)\right)\right) \quad x \mapsto \kappa\left(f_{L}(\iota(x))\right) \text {. }
\end{aligned}
$$

PROPOSITION 3.1.7. Consider the following diagram: 


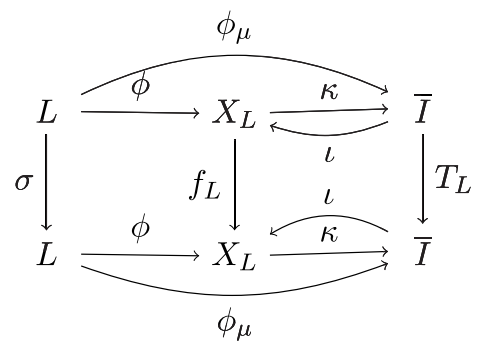

(a) for every $x \in I, \iota\left(T_{L}(x)\right)=f_{L}(\iota(x))$;

(b) $(L, \sigma)$ is topologically conjugate to $\left(X_{L}, f_{L}\right)$ by $\phi$;

(c) $\left(\left(\iota(\bar{I}) \subset X_{L}\right), f_{L}\right)$ is topologically semi-conjugate to $\left(\bar{I}, T_{L}\right)$ by $\kappa$;

(d) $\left(\left(\phi^{-1}(\iota(\bar{I})) \subset L\right), \sigma\right)$ is topologically semi-conjugate to $\left(\bar{I}, T_{L}\right)$ by $\phi_{\mu}$.

Proof. (a) Note that $0,1 \notin Z_{0}$. Indeed, $\phi_{\mu}$ being non-decreasing, if $\phi_{\mu}^{-1}(0)$ (respectively $\phi_{\mu}^{-1}(1)$ ) was made of two words, consecutive by Lemma 3.1.5, they would be the two smallest (respectively greatest) in $L$, and by Lemma 3.1.4 the smaller one (respectively greater one) would be isolated, contradicting Remark 3.1.1. Now, we put $I^{\prime}=\bar{I} \backslash\left(Z_{0} \cup\{0,1\}\right)$. By definition, $\kappa$ is injective on $\iota(I)$, hence on $\iota\left(I^{\prime}\right)$, thus since $\kappa$ is a left inverse of $\iota$, it is also a right inverse of $\iota$ when restricted to this set. We compose $T_{L}=\kappa \circ f_{L} \circ \iota$ on both sides to the left by $\iota$, so that $\iota \circ T_{L}=f_{L} \circ \iota$ is valid on $I^{\prime}$, provided that $f_{L}\left(\iota\left(I^{\prime}\right)\right) \subset \iota\left(I^{\prime}\right)$, which is proved as follows: by definition of $I^{\prime}$, for every $x \in I^{\prime}$, $\phi_{\mu}^{-1}(x)=\phi^{-1}(\iota(x))$, that is, $\phi_{\mu}^{-1}\left(I^{\prime}\right)=\phi^{-1}\left(\iota\left(I^{\prime}\right)\right)$. Also, by Lemmas 3.1.4 and 3.1.5, a word $w$ is in $\phi_{\mu}^{-1}\left(I^{\prime}\right)$ if and only if it is not a cylinder endpoint. Thus, there are words in $L$ arbitrarily close to $w$ from the right and the left, that is, words smaller and greater than $w$ with arbitrarily long prefixes common with those of $w$. By continuity of $\sigma$, the same holds for $\sigma(w)$, so $\sigma\left(\phi^{-1}\left(\iota\left(I^{\prime}\right)\right)\right) \subset \phi^{-1}\left(\iota\left(I^{\prime}\right)\right)$. Composing on both sides to the left by $\phi$ yields $f_{L}\left(\iota\left(I^{\prime}\right)\right) \subset \iota\left(I^{\prime}\right)$, as required. Now, $Z_{0}$ being countable, $I^{\prime}$ is dense in $\bar{I}$. Since both $\iota \circ T_{L}$ and $f_{L} \circ \iota$ are compositions of right-continuous maps on $I$, they are right-continuous too, so $\iota \circ T_{L}=f_{L} \circ \iota$ extends to all of $I$.

(b) $\phi$ is a homeomorphism, thus by definition of $f_{L}$, the result follows.

(c) By definition of $T_{L}$, we have the conjugacy $T_{L} \circ \kappa=\kappa \circ f_{L}$ on $\iota\left(I^{\prime}\right)$. Both members of this identity are right-continuous maps, and by the same arguments as in (a), this conjugacy holds on all of $\iota(I)$. Next, again by definition of $T_{L}$, we have $T_{L}(1)=$ $\kappa\left(f_{L}(\iota(1))\right)$, and 1 has only one preimage by $\kappa$ which is $\iota(1)$, so the conjugacy holds on $\iota(\bar{I})$ too. Finally, $\kappa$ is measure-preserving and onto.

(d) Using the fact that $\kappa \circ \phi=\phi_{\mu}$, and the commutativity properties given by (b) and (c) on $\kappa$ and $\phi$, the result follows.

3.2. Checking the infinite exchange transformation properties. We now focus on when $T_{L}$ is an IET. Here are first three general lemmas.

LEMMA 3.2.1. The image of $\mu$ by $\phi_{\mu}$ is the Lebesgue measure on $\bar{I}$, which is preserved by $T_{L}$. 
Proof. We denote by $f_{*} \mu$ the image of the measure $\mu$ by the map $f$. By Lemmas 3.1.2 and 3.1.3, $\phi_{\mu}$ is continuous and surjective, thus the preimage of any interval $[0, x]$, with $x \in \bar{I}$, is an interval $\left[w_{L, \min }, w\right]$ in $L$, where $\phi_{\mu}(w)=x=\mu\left(\left[w_{L, \min }, w\right]\right)$. Hence $\left(\phi_{\mu}\right)_{*} \mu$ is the Lebesgue measure on $\bar{I}$. Next, by Proposition 3.1.7(d), $\phi_{\mu} \circ \sigma=T_{L} \circ \phi_{\mu}$ on $\phi^{-1}(\iota(\bar{I}))$, a set of full measure in $L$. Thus $\left(\phi_{\mu}\right)_{*} \sigma_{*} \mu=T_{L *}\left(\phi_{\mu}\right)_{*} \mu$, and since $\sigma$ preserves $\mu,\left(\phi_{\mu}\right)_{*} \sigma_{*} \mu=\left(\phi_{\mu}\right)_{*} \mu$, that is, $T_{L}$ preserves the Lebesgue measure on $\bar{I}$.

From $\mathrm{CYL}_{L}(1)$, that is, the partition of $L$ defined as $\bigsqcup_{a \in A} \mathrm{Cyl}_{L}(a)$, we define the induced partition of $I$ as

$$
P_{A}: I=\bigsqcup_{a \in A} I_{L, a}, \quad \text { where } I_{L, a}=\left\{x \in I \mid \phi^{-1}(\iota(x)) \in \mathrm{Cyl}_{L}(a)\right\} .
$$

Note that each $I_{L, a}$ is a right-open interval in $I$. Indeed, if $x<x^{\prime}$, with $x, x^{\prime} \in I_{L, a}$, then every $x^{\prime \prime} \in I$, with $x<x^{\prime \prime}<x^{\prime}$, is also in $I_{L, a}$ since $\phi^{-1}$ and $\iota$ both preserve the order. Moreover, if $a<a^{\prime}$, where $a, a^{\prime} \in A$ are consecutive, then if $w$ is the greatest word in $\operatorname{Cyl}_{L}(a)$ and $w^{\prime}$ the smallest in $\operatorname{Cyl}_{L}\left(a^{\prime}\right)$, we have $\phi_{\mu}(w)=\phi_{\mu}\left(w^{\prime}\right)=y$, where $y \in Z_{0}$. By definition of $Z_{0}$ and $\phi$ and since $w<w^{\prime}, \phi^{-1}(\iota(y))=w^{\prime}$, thus $y \in I_{L, a^{\prime}}$, which means that $I_{L, a}$ is right-open.

LeMma 3.2.2. $T_{L}$ is right-continuous on $I$, and piecewise increasing on $P_{A}$.

Proof. $T_{L}$ is right-continuous on $I$, being a composition of the right-continuous maps $\kappa, f_{L}$, and $\iota$. Next, $T_{L}$ is non-decreasing on each $I_{L, a}$ in $P_{A}$. Indeed, by Proposition 3.1.7(d), $T_{L} \circ \phi_{\mu}=\phi_{\mu} \circ \sigma$ on $\phi^{-1}\left(\iota\left(I_{L, a}\right)\right) \subset \mathrm{Cyl}_{L}(a)$, and $\phi_{\mu}$ is nondecreasing on $I$, while $\sigma$ is increasing on $\operatorname{Cyl}_{L}(a)$. Now, assume there exist $x, x^{\prime} \in I_{L, a}$, with $x<x^{\prime}$, such that $T_{L}(x)=T_{L}\left(x^{\prime}\right)$. Since $T_{L}$ is non-decreasing it must be constant on ( $\left.x, x^{\prime}\right)$, which is impossible since $T_{L}$ is measure-preserving by Lemma 3.2.1, hence $T_{L}$ is increasing on each $I_{L, a}$.

LEMMA 3.2.3. $T_{L}(I) \subset I$.

Proof. By Lemma 3.2.2, $T_{L}$ is piecewise increasing on the $I_{L, a}$. Let $J$ be one of these intervals, and assume $T_{L}(x)=1$ for some $x \in J$. But with $T_{L}$ being increasing on $J$, and $J$ being right-open, $T_{L}(y)$ would be $>1$ for all $y \in J$ with $y>x$.

As a next step, let us exhibit conditions so that there exists a partition of $I$ on which $T_{L}$ is a translation on its components $\left(P_{A}\right.$ is not such a partition in general). For that purpose, we first study the effect of $\sigma$ over the word intervals in $L$. A factor $u \in$ Fact $_{L}$ is said to be left special (respectively right special) if $u$ has at least two distinct left (respectively right) letter prolongations in $\operatorname{Fact}_{L}$, i.e. if $u \in \operatorname{Fact}_{L}(n)$, then $u$ is the suffix (respectively prefix) of at least two distinct factors in $\operatorname{Fact}_{L}(n+1)$.

LEMMA 3.2.4. Let $L$ be a shift such that $\mathrm{Fact}_{L}$ is prolongable. Let $w, w^{\prime} \in \mathrm{Cyl}_{L}(a u)$, with $a \in A, u \in$ Fact $_{L}$ not left special, and $w<w^{\prime}$. Then

$$
\sigma\left(\left[w, w^{\prime}\right]\right)=\left[\sigma(w), \sigma\left(w^{\prime}\right)\right] .
$$


Proof. (ㄷ) Let $w=a u v$ and $w^{\prime}=a u v^{\prime}$, with $v, v^{\prime} \in A^{\mathbb{N}}$. Let $w^{\prime \prime}=a u v^{\prime \prime} \in\left[w, w^{\prime}\right]$, with $v^{\prime \prime} \in A^{\mathbb{N}}$. By lexicographic order, we have $u v \leq u v^{\prime \prime} \leq u v^{\prime}$. Hence $\sigma\left(w^{\prime \prime}\right)=u v^{\prime \prime}$ belongs to $\left[u v, u v^{\prime}\right]=\left[\sigma(w), \sigma\left(w^{\prime}\right)\right]$.

() Let again $w=a u v$ and $w^{\prime}=a u v^{\prime}$, with $v, v^{\prime} \in A^{\mathbb{N}}$. Let $w^{\prime \prime}=u v^{\prime \prime} \in$ $\left[\sigma(w), \sigma\left(w^{\prime}\right)\right]$, with $v^{\prime \prime} \in A^{\mathbb{N}}$. Consider $\left\{u u_{j}\right\}_{j \in \mathbb{N}}$ the sequence of all the prefixes of $w^{\prime \prime}$ with $u_{j} \in A^{*}$ starting with $u$. By prolongability to the left, each $u u_{j}$ has at least one left letter prolongation, but it can only be $a$ since $u$ is not left special. By prolongability to the right, each $a u u_{j}$ is the prefix of at least one word in $L$, which makes a sequence converging to the word $a w^{\prime \prime}$, belonging to $L$ by compactness of $L$. Thus there is one left letter prolongation for $w^{\prime \prime}$ in $L$, unique since $u$ is not left special, that is, $a w^{\prime \prime}=\sigma^{-1}\left(w^{\prime \prime}\right)=$ $\sigma^{-1}\left(u v^{\prime \prime}\right)$. Since $u v \leq u v^{\prime \prime} \leq u v^{\prime}$, we have $a u v \leq a w^{\prime \prime} \leq a u v^{\prime}$, that is, $a w^{\prime \prime} \in\left[w, w^{\prime}\right]$. Hence $w^{\prime \prime} \in \sigma\left(\left[w, w^{\prime}\right]\right)$.

Note that a specific case of the above is $\sigma\left(\operatorname{Cyl}_{L}(a u)\right)=\operatorname{Cyl}_{L}(u)$, where $a \in A$ and $u$ is not left special. Now, the idea is to build a partition of $L$ essentially made of cylinders such that equality (2) holds in all of these cylinders. An infinite left special word (or infinite left special branch) with respect to Fact $L$, where $L \subseteq A^{\mathbb{N}}$, is a one-way infinite word such that all its prefixes are left special factors in $\mathrm{Fact}_{L}$. We denote by $\mathrm{SP}_{L} \subseteq A^{\mathbb{N}}$ the set of all the infinite left special words with respect to Fact $_{L}$. When $L$ is a shift, $\mathrm{SP}_{L}$ is included in $L$.

LEMMA 3.2.5. Let L be a shift having left special factors of arbitrary length, measured by $\mu$, with $\mu\left(\mathrm{SP}_{L}\right)=0$. Then, there is an infinite partition of $L$ defined as

$$
\operatorname{PART}_{L}: L=\bigsqcup_{k>0} \operatorname{Cyl}_{L}\left(v^{(k)}\right) \sqcup W_{\mathrm{SP}_{L}},
$$

where:

- for each $k>0, v^{(k)} \in \mathrm{Fact}_{L}$, such that for all $w, w^{\prime} \in \mathrm{Cyl}_{L}\left(v^{(k)}\right)$ with $w<w^{\prime}$, $\sigma\left(\left[w, w^{\prime}\right]\right)=\left[\sigma(w), \sigma\left(w^{\prime}\right)\right]$ (i.e. equality (2) holds).

- $W_{\mathrm{SP}_{L}}=\sigma^{-1}\left(\mathrm{SP}_{L}\right)$ is a null measure non-empty set in $L$, which is formed by the accumulation of the endpoints of $\mathrm{Cyl}_{L}\left(v^{(k)}\right)$ in $L$.

Proof. Let us describe the cylinders of $\mathrm{PART}_{L}$ by an iterative process. As a first step, consider the partition $\mathrm{CYL}_{L}(2)$ of $L$, i.e. $\bigsqcup_{a_{i} a_{j} \in \operatorname{Fact}_{L}(2)} \mathrm{Cyl}_{L}\left(a_{i} a_{j}\right)$, with $a_{i}, a_{j} \in A$. By Lemma 3.2.4, for each non-left special $a_{j}$, we have $\sigma\left(\left[w, w^{\prime}\right]\right)=\left[\sigma(w), \sigma\left(w^{\prime}\right)\right]$ for all $w, w^{\prime} \in \operatorname{Cyl}_{L}\left(a_{i} a_{j}\right)$, so that $\operatorname{Cyl}_{L}\left(a_{i} a_{j}\right)$ is put in $\operatorname{PART}_{L}$, and $a_{i} a_{j}$ is one of the $v^{(k)}$. As a second step, each of the remaining $\operatorname{Cyl}_{L}\left(a_{i} a_{j}\right)$ not put in $\mathrm{PART}_{L}$ during the first step, is partitioned with cylinders of the form $\operatorname{Cyl}_{L}\left(a_{i} a_{j} a_{k}\right)$ in $\mathrm{CYL}_{L}(3)$. Again, for each non-left special suffix $a_{j} a_{k}$, the word intervals in $\mathrm{Cyl}_{L}\left(a_{i} a_{j} a_{k}\right)$ satisfy equality (2), and $\operatorname{Cyl}_{L}\left(a_{i} a_{j} a_{k}\right)$ is put in $\mathrm{PART}_{L}$, while $a_{i} a_{j} a_{k}$ is also one of the $v^{(k)}$. This refinement process is inductively applied as long as cylinders remain at step $n$ by partitioning them with cylinders in $\mathrm{CYL}_{L}(n+1)$, defining then all the cylinders of $\mathrm{PART}_{L}$.

Since the left special factors of $L$ can have arbitrary lengths, and since every prefix of a left special word is left special, there is at least one left special factor in $L$ for each length. Thus, given any $n>0$ and a left special factor $u \in \operatorname{Fact}_{L}(n)$, the cylinder $\operatorname{Cyl}_{L}(a u), a \in A$, must still be refined during the $n$th step of the above refinement process. Therefore, this process is necessarily infinite. It determines infinite sequences of nested cylinders of the 
form $\left\{\operatorname{Cyl}_{L}\left(a u_{j}\right)\right\}_{j \in \mathbb{N}^{*}}$, where $u_{j} \in \operatorname{Fact}_{L}(j)$ is left special while being a prefix of $u_{j+1}$, for all $j$. Such a sequence $\left\{a u_{j}\right\}_{j \in \mathbb{N}^{*}}$ gives a limit infinite word, which belongs to $W_{\mathrm{SP}_{L}}$ since $\left\{u_{j}\right\}_{j \in \mathbb{N}^{*}}$ gives a limit infinite word in $\mathrm{SP}_{L}$. As a result, if a word $w \in L$ does not belong to some $\operatorname{Cyl}_{L}\left(v^{(k)}\right)$, it belongs to $W_{\mathrm{SP}_{L}}$. Conversely, if $w \in \mathrm{SP}_{L}$, there exist more than one left letter prolongation to each of its prefixes. Consider any of these letters, say $a \in A$, so that $a w \in \sigma^{-1}\left(\mathrm{SP}_{L}\right)=W_{\mathrm{SP}_{L}}$. According to the same above argument, each prefix $u_{j}$ of $w$ determines a cylinder $\operatorname{Cyl}_{L}\left(a u_{j}\right)$ which has to be refined further. We have $\bigcap_{u_{j}}$ prefix of ${ }_{w} \operatorname{Cyl}_{L}\left(a u_{j}\right)=a w$, where $a w$ does not belong to any cylinder $\operatorname{Cyl}_{L}\left(v^{(k)}\right)$, and induces an infinite refinement. Thus PART $_{L}$ is a partition since a word $w$ in $L$ belongs either to one of the cylinders $\mathrm{Cyl}_{L}\left(v^{(k)}\right)$ obtained through a finite number of steps in the refinement process, or to $W_{\mathrm{SP}_{L}}$ if $w$ induces an infinite number of steps.

The set $W_{\mathrm{SP}_{L}}$ is not empty since $\mathrm{SP}_{L}$ is not empty when there are infinitely many left special factors. Also, $W_{\mathrm{SP}_{L}}$ is of null measure since $\mathrm{SP}_{L}$ has been assumed of null measure and $\sigma$ is measure-preserving. Finally, $W_{\mathrm{SP}_{L}}$ occurs as the set of the accumulation of the $\mathrm{Cyl}_{L}\left(v^{(k)}\right)$ endpoints, since again $\mathrm{SP}_{L}$ is of null measure, thus there is no cylinder having all its words in $\mathrm{SP}_{L}$.

Note that if $L$ is minimal aperiodic, it has left special factors of arbitrary length.

In order to exhibit examples of aperiodic minimal shifts, we use the following classic technique. A substitution is a map $\theta: A \rightarrow A^{\prime *}$, where $A$ and $A^{\prime}$ are alphabets, which is extended to words by sending $w=\ldots a_{i} a_{i+1} a_{i+2} \ldots$ to $\theta(w)=$ $\ldots \theta\left(a_{i}\right) \theta\left(a_{i+1}\right) \theta\left(a_{i+2}\right) \ldots$, that is, on finite words, $\theta$ is just a monoid morphism from $A^{*}$ to $A^{\prime *}$. For instance, the Thue-Morse substitution $\theta_{t m}$ over $A=A^{\prime}=\{a, b\}$ is defined by $\theta_{t m}(a)=a b$ and $\theta_{t m}(b)=b a$. When $A=A^{\prime}$, a substitution can be iterated. The set of factors of such a substitution is defined as $\operatorname{Fact}_{\theta}=\left\{v \in A^{*} \mid v \in \operatorname{Fact}_{\theta^{n}}(a), a \in A, n \in\right.$ $\mathbb{N}\}$, and its associated shift as $L_{\theta}=\left\{w \in A^{\mathbb{N}} \mid\right.$ Fact $_{w} \subseteq$ Fact $\left._{\theta}\right\}$. When $\theta$ is non-erasing, i.e. there is no $a \in A$ whose image is the empty word, and when $a$ is a strict prefix of $\theta(a)$, then for all $n \geq 0, \theta^{n}(a)$ is a strict prefix of $\theta^{(n+1)}(a)$. Thus $\left\{\theta^{n}(a)\right\}_{n \in \mathbb{N}}$ gives rise to a limit word in $A^{\mathbb{N}}$, denoted by $\theta^{\omega}(a)$, which is a fixed point of $\theta$. For instance, the ThueMorse substitution has two fixed points: $\theta_{t m}^{\omega}(a)=w_{1}=a b b a b a a b b a a b a b b a \ldots$ and $\theta_{t m}^{\omega}(b)=w_{2}=$ baababbaabbabaab . . . Considering a fixed point $w$ of a substitution $\theta$, its associated shift $L_{\theta, w}$ is then defined as the closure of $\left\{\sigma^{n}(w) \mid n \in \mathbb{N}\right\}$. A substitution $\theta$ is said to be primitive if there exists $n>0$ such that for every $a, b \in A$, the word $\theta^{n}(a)$ contains $b$. For a primitive substitution $\theta$, it is known that [Qué10]: (1) if $w$ is any of its fixed points, $L_{\theta}=L_{\theta, w}$; (2) for all $n>0, L_{\theta^{n}}=L_{\theta}$, so that periodic points can also be considered; (3) $L_{\theta}$ is minimal. Thus for instance the Thue-Morse substitution $\theta_{t m}$ generates a minimal shift $L_{t m}$, equal to $L_{\theta_{t m}}=L_{\theta_{t m}, w_{1}}=L_{\theta_{t m}, w_{2}}$. A primitive substitution is said to be aperiodic if its associated shift is minimal aperiodic, e.g. $\theta_{t m}$ is known to be aperiodic [Thu12]†.

Example 3.2.6. (Step-by-step construction of a partition $\mathrm{PART}_{L}$ ) Considering the above Thue-Morse shift $L_{t m}$ and its fixed points $w_{1}, w_{2}$, we build here the first components of

$†$ More generally, aperiodicity for a substitution is decidable using [ER83]. 
$\operatorname{PART}_{L_{t m}}$ as in the proof of Lemma 3.2.5. The first factor sets are:

$$
\begin{aligned}
& \text { Fact }_{L_{t m}}(1)=\{a, b\} \\
& \text { Fact }_{L_{t m}}(2)=\{a a, a b, b a, b b\} \\
& \text { Fact }_{L_{t m}}(3)=\{a a b, a b a, a b b, b a a, b a b, b b a\} \\
& \text { Fact }_{L_{t m}}(4)=\{a a b a, a a b b, a b a a, a b a b, a b b a, b a a b, b a b a, b a b b, b b a a\} \\
& \text { Fact }_{L_{t m}}(5)=\{a a b a b, a a b b a, a b a a b, a b a b b, a b b a a, a b b a b, b a a b a, b a a b b \text {, } \\
& \text { babaa, babba, bbaab, bbaba\}. }
\end{aligned}
$$

Thus its first left special factors are: $a, b, a b, b a, a b a, a b b, b a a, b a b, a b b a, b a a b$. Applying the refinement process, the first step, based on $\mathrm{CYL}_{L}(2)$, gives no cylinders of PART $_{L_{t m}}$ since $a$ and $b$ are both left special factors. The second step, based on $\mathrm{CYL}_{L}(3)$, gives $\mathrm{Cyl}_{L_{t m}}(a b b)$ and $\mathrm{Cyl}_{L_{t m}}(b a a)$, since $a a$ and $b b$ are not left special factors, thus $v^{(1)}=a b b$ and $v^{(2)}=b a a$. The third step gives no new cylinders since $a b b a$ and $b a a b$ are the only factors whose right suffix is not left special, already contained in the cylinders of the preceding step. The fourth step gives $\mathrm{Cyl}_{L_{t m}}(a a b a b), \mathrm{Cyl}_{L_{t m}}(a b a b b), \mathrm{Cyl}_{L_{t m}}($ babaa $)$, and $\mathrm{Cyl}_{L_{t m}}(b b a b a)$, thus $v^{(3)}=a a b a b, v^{(4)}=a b a b b, v^{(5)}=b a b a a$, and $v^{(6)}=b b a b a$. And so on.

Now, given a shift $L$ and PART $_{L}$ as obtained from Lemma 3.2.5, we define the induced partition of $I$ as follows:

$$
\operatorname{PART}_{L, I}: I=\bigsqcup_{k>0} I_{L}^{(k)} \sqcup B_{\mathrm{SP}_{L}},
$$

where $I_{L}^{(k)}=\left\{x \in I \mid \phi^{-1}(\iota(x)) \in \mathrm{Cyl}_{L}\left(v^{(k)}\right)\right\}$ and $B_{\mathrm{SP}_{L}}=\left\{x \in I \mid \phi^{-1}(\iota(x)) \in W_{\mathrm{SP}_{L}}\right\}$. Each $I_{L}^{(k)}$ is a right-open interval by the same arguments as for the intervals of the partition $P_{A}$ (see p. 1943).

Let $B_{L}$ be the closure in $\bar{I}$ of the left ends of the $I_{L}^{(k)}$, intersected with $I$ (so that $1 \notin B_{L}$ ). Let $B_{L, \text { acc }}$ (respectively $B_{L, \text { acc }, r}$ ) be the set of accumulation points (respectively accumulation points from the right) of $B_{L}$ in $\bar{I}$. Note that $B_{\mathrm{SP}_{L}} \subset B_{L \text {, acc }}$ and even that $B_{\mathrm{SP}_{L}}=B_{L, \text { acc, } r}$ since an accumulation point $x$ only to the left is the left endpoint of some $I_{L}^{(k)}$ and does not belong to $B_{\mathrm{SP}_{L}}$ (in this case, $\phi^{-1}(\iota(x)) \notin W_{\mathrm{SP}_{L}}$ because of the differences between $\iota(I)$ and $X_{L}$-see also Definition 2).

LEMMA 3.2.7. $B_{L} \sqcup\{1\}$ is a null-measure infinite compact set of points in $\bar{I}$.

Proof. The set $B_{L} \sqcup\{1\}$ is compact by definition. Since the set of the left endpoints of the $I_{L}^{(k)}$ is countable, the measure of $B_{L}$ is the same as that of $B_{L \text {, acc }}$, and also as that of $B_{L, \text { acc, } r}$ which is equal to $B_{\mathrm{SP}_{L}}$. Now, recall from Lemma 3.2.1 that the Lebesgue measure is the image of $\mu$ by $\phi_{\mu}$. Since $\phi_{\mu}^{-1}\left(B_{\mathrm{SP}_{L}}\right)=W_{\mathrm{SP}_{L}}$ and $\mu\left(W_{\mathrm{SP}_{L}}\right)=0$, the measure of $B_{L}$ is also zero in $\bar{I}$.

LEMMA 3.2.8. $T_{L}$ is a translation on each interval component $I_{L}^{(k)}$ of $\operatorname{PART}_{L, I}$.

Proof. Let the interior of $I_{L}^{(k)}$ be denoted by $I_{L}^{(k)^{o}}$. By definition of $T_{L}$, for all $x \in I_{L}^{(k)^{o}}$,

$$
T_{L}(x)-x=\kappa\left(\phi\left(\sigma\left(\phi^{-1}(\iota(x))\right)\right)\right)-x .
$$


We first put $\phi^{-1}(\iota(x))=w$. Next, recalling that $\phi_{\mu}=\kappa \circ \phi$ and that $\kappa \circ \iota$ is the identity, we compose $\phi^{-1}(\iota(x))=w$ on both sides to the left by $\kappa \circ \phi$ and get $x=\kappa(\phi(w))=\phi_{\mu}(w)$, so that (5) becomes for all $x \in I_{L}^{(k)^{o}}$,

$$
T_{L}(x)-x=\kappa(\phi(\sigma(w)))-\kappa(\phi(w))=\phi_{\mu}(\sigma(w))-\phi_{\mu}(w) .
$$

By definition of $\operatorname{PART}_{L, I}$, the inverse image of $I_{L}^{(k)^{o}}$ by $\phi_{\mu}$ is the interior $C^{(k)^{o}}$, where $C^{(k)}$ denotes $\operatorname{Cyl}_{L}\left(v^{(k)}\right)$ of $\mathrm{PART}_{L}$. As a closed ordered set, $C^{(k)}$ has a smallest word $w_{C^{(k)} \text {,min }}$, and recall that $w_{L, \text { min }}$ denotes the smallest word of $L$, so that for all $w \in C^{(k)^{o}}$, $\left[w_{L, \min }, w\right]=\left[w_{L, \min }, w_{C^{(k)}, \min }\right] \sqcup\left(w_{C^{(k)}, \min }, w\right]$, then

$$
\phi_{\mu}(w)=\mu\left(\left[w_{L, \min }, w_{C^{(k)}, \min }\right]\right)+\mu\left(\left[w_{C^{(k)}, \min }, w\right]\right) .
$$

Since $w_{C^{(k)}}$,min and $w$ belong to the same cylinder, and $w_{C^{(k)} \text {, min }}<w$, we have $\sigma\left(w_{C^{(k)}, \min }\right)<\sigma(w)$, hence

$$
\phi_{\mu}(\sigma(w))=\mu\left(\left[w_{L, \min }, \sigma\left(w_{C^{(k)}, \min }\right)\right]\right)+\mu\left(\left[\sigma\left(w_{C^{(k)}, \min }\right), \sigma(w)\right]\right) .
$$

Next, using the properties over the components of $\operatorname{PART}_{L}$,

$$
\begin{aligned}
\phi_{\mu}(\sigma(w)) & \left.=\mu\left(\left[w_{L, \min }, \sigma\left(w_{C^{(k)}, \min }\right)\right]\right)+\mu\left(\sigma\left(\left[w_{C^{(k)}, \min }, w\right]\right)\right) \quad \text { (by equality }(2)\right) \\
& =\mu\left(\left[w_{L, \min }, \sigma\left(w_{C^{(k)}, \min }\right)\right]\right)+\mu\left(\sigma^{-1} \sigma\left(\left[w_{C^{(k)}, \min }, w\right]\right)\right) \quad(\mu \text {-preservation }) \\
& =\mu\left(\left[w_{L, \min }, \sigma\left(w_{C^{(k)}, \min }\right)\right]\right)+\mu\left(\left[w_{C^{(k)}, \min }, w\right]\right) \quad\left(\sigma \text { injectivity on } C^{(k)^{o}}\right) .
\end{aligned}
$$

Therefore, from the above last equation and (7), we get for all $w \in C^{(k)^{o}}$,

$$
\phi_{\mu}(\sigma(w))-\phi_{\mu}(w)=\mu\left(\left[w_{L, \min }, \sigma\left(w_{C^{(k)}, \min }\right)\right]\right)-\mu\left(\left[w_{L, \min }, w_{C^{(k)}, \min }\right]\right)=K_{C^{(k)}} .
$$

Thus $K_{C^{(k)}}$ depends only on the $C^{(k)^{o}}$ to which $w$ belongs, that is, it depends only on the open interval $I_{L}^{(k)^{o}}$ to which $x$ belongs. Hence, for every $I_{L}^{(k)}$ of $\operatorname{PART}_{L, I}$, and its corresponding cylinder $C^{(k)}=\mathrm{Cyl}_{L}\left(v^{(k)}\right)$ of $\mathrm{PART}_{L}$, there is a constant $K_{C^{(k)}}$ such that for all $x \in I_{L}^{(k)^{o}}, T_{L}(x)-x=K_{C^{(k)}}$. By right-continuity of $T_{L}$ (see Lemma 3.2.2), this equality extends to all of $I_{L}^{(k)}$.

Following Definition 2, let $\mathcal{D}_{L}$ be the closure of $Y_{L}$ in $I$, where $Y_{L}$ is the set of discontinuities of $T_{L}$, which by Lemma 3.2.8 is necessarily included into $B_{L}$. Let $\mathcal{D}_{L}$, acc, $r$ be the set of accumulation points of $\mathcal{D}_{L}$ from the right.

LeMma 3.2.9. $T_{L}$ is injective on $I \backslash \mathcal{D}_{L, \text { acc }, r}$.

Proof. By Lemma 3.2.8, $T_{L}$ is a translation on each of the right-open interval components $I_{L}^{(k)}$ of PART $_{L, I}$. The set $\mathcal{D}_{L}$ can be obtained by dropping all the points from $B_{L}$ having a neighborhood where $T_{L}$ extends as a translation map, so that $T_{L}$ is still a translation map on each right-open interval component of $I \backslash \mathcal{D}_{L}$. By Lemma 3.2.1, the Lebesgue measure is preserved by $T_{L}$, and by Lemma 3.2.7, $B_{L}$ has null measure, thus $\mathcal{D}_{L} \subseteq B_{L}$ has too. Now, if the open interval components of $I \backslash \mathcal{D}_{L}$ had overlaps in their translated images by $T_{L}$, the global image would be a set $I^{\prime}$ with $\mu\left(I^{\prime}\right)<1$, such that $\mu\left(T^{-1}\left(I^{\prime}\right)\right)=$ $\mu\left(I \backslash \mathcal{D}_{L}\right)=1$, contradicting measure preservation. Moreover, no image by $T_{L}$ of a left endpoint of these open intervals can lie in the image of another interval, thus $T$ is injective on all the corresponding right-open intervals. Since $\mathcal{D}_{L} \backslash \mathcal{D}_{L, \text { acc, } r}$ consists of all these left endpoints, the result follows. 
LEMMA 3.2.10. Let $L$ be a shift measured by $\mu$. If $L$ has zero topological entropy, then $\mu\left(\mathrm{SP}_{L}\right)=0$.

Proof. The topological entropy dominates the (measure-theoretic) entropies on $L$ with respect to all the invariant probability measures [HK02, Proposition 4.4.1]. Thus with respect to $\mu$, the shift $L$ has necessarily zero entropy. But then, a dynamical system with zero entropy with respect to an invariant measure is known to be invertible [HK02, Section $3.7 \mathrm{k}$ ], that is, it has an inverse on a subset of full measure. In the case of a shift $L$, the set of words on which the shift map is not invertible is exactly $\mathrm{SP}_{L}$, so that $\mu\left(\mathrm{SP}_{L}\right)=0$.

We are now ready to prove Theorem 1 as stated in the introduction.

Proof. Since $L$ is assumed aperiodic minimal, the results of $\S 3.1$ apply, so that $(L, \sigma)$ is a topological conjugate by $\phi$ of $\left(X_{L}, f_{L}\right)$, where $I$ is embedded as a subset of $X_{L}$ in such a way that $f_{L}$ is an extension of $T_{L}$. By Lemma 3.2.2, $T_{L}$ is right-continuous, and by Lemma 3.2.10, $\mu\left(\mathrm{SP}_{L}\right)=0$ for any $\sigma$-invariant Borel probability measure $\mu$ chosen for $L$. Minimal aperiodicity means $L$ has left special factors of arbitrary length, thus Lemma 3.2.5 applies. Hence, by Lemmas 3.2.7-3.2.9, and since the set $\mathcal{D}_{L}$ of $T_{L}$ is included into $B_{L}$, $T_{L}$ is an IET.

Note that Theorem 1 remains valid under the assumptions on $L$ in Lemma 3.2.5, in particular $\mu\left(\mathrm{SP}_{L}\right)=0$ can be used instead of zero topological entropy. Note also that the above construction relies on $B_{L}$, a set determined from PART $_{L, I}$, determined in turn from PART $_{L}$. This set of separation points for $T_{L}$ could have been different, as it is just a set with the property of including $\mathcal{D}_{L}$ (see $\$ 2.1$ ). However, following the construction of $\mathrm{PART}_{L}$ in the proof of Lemma 3.2.5, this set has some word-combinatorics properties, and we shall use them henceforth.

As a complement, in view of Definition 2 of an infinite IET and of Lemma 3.2.9, we give the following example.

Example 3.2.11. ( $T_{L}$ can be non-injective on $\left.\mathcal{D}_{L, \text { acc }, r}\right)$ Let us reconsider the Thue-Morse substitution $\theta_{t m}$ over $A=\{a, b\}$ (see Example 3.2.6), its associated shift $L_{t m}$, and its fixed points $w_{1}=\theta_{t m}^{\omega}(a)$ and $w_{2}=\theta_{t m}^{\omega}(b)$. A bispecial factor is a factor which is both right and left special. According to [Cas96], the bispecial factors in $L_{t m}$ are $\theta_{t m}^{n}(a), \theta_{t m}^{n}(b)$, $\theta_{t m}^{n}(a b a), \theta_{t m}^{n}(b a b)$, with $n \geq 0$. Thus we have $\left\{w_{1}, w_{2}\right\}=\mathrm{SP}_{L_{t m}}$. In fact, in an aperiodic minimal shift, every infinite special word has always infinitely many prefixes which are bispecial factors.

Let the lexicographic order over $L_{t m}$ be determined by $a<b$. We claim that $w_{1}, w_{2}$ are consecutive in $L_{t m}$, with $w_{1}$ being the greatest word in $\operatorname{Cyl}_{L_{t m}}(a)$, and $w_{2}$ the smallest word in $\mathrm{Cyl}_{L_{t m}}(b)$. Assume on the contrary there is $w_{1}^{\prime} \in \mathrm{Cyl}_{L_{t m}}(a)$ with $w_{1}<w_{1}^{\prime}$. Let $u$ be the maximal common prefix so that $w_{1}=u v_{1}$ and $w_{1}^{\prime}=u v_{1}^{\prime}$. Then $u$ is bispecial, since at least three of the factors $a u a, a u b, b u a, b u b$ are in Fact $_{L_{t m}}$ (both $u a$ and $u b$ are prolongable to the left, and at least one of them is left special, being a prefix of $w_{1}$ ). By [Cas96], such a bispecial factor in $L_{t m}$ with at least three continuation factors and starting by $a$ must be of the form $\theta_{t m}^{n}(a)$ for some $n \geq 0$. Now, since $w_{1}<w_{1}^{\prime}$, the word $v_{1}$ 
must also start with $a$, and $v_{1}^{\prime}$ with $b$. However, since there is $n>0$ such that $u=\theta_{t m}^{n}(a)$ and since $\theta_{t m}^{n+1}(a)=\theta_{t m}^{n}(a) \theta_{t m}^{n}(b)$ is also a prefix of $w_{1}$, then $u$ must be followed by $b$ in $w_{1}$. Hence there is no such word as $w_{1}^{\prime}$ and $w_{1}$ is the greatest word in $\operatorname{Cyl}_{L_{t m}}(a)$. Similar arguments apply to $w_{2}$ showing it is the smallest word in $\mathrm{Cyl}_{L_{t m}}(b)$. Thus by Lemma 3.1.4, $w_{1}, w_{2}$ are consecutive.

As a result, $\left\{a w_{1}, a w_{2}\right\}$ and $\left\{b w_{1}, b w_{2}\right\}$ are two pairs of consecutive words in $L_{t m}$. Moreover, $\mathrm{Cyl}_{L_{t m}}(a b b)$ is a non-empty cylinder between $a w_{2}$ and $b w_{1}$, then by Lemmas 3.1.2 and 3.1.5, $\phi_{\mu}\left(a w_{1}\right)=\phi_{\mu}\left(a w_{2}\right)<\phi_{\mu}\left(b w_{1}\right)=\phi_{\mu}\left(b w_{2}\right)$. Now, since $L_{t m}$ has linear complexity [Cas96], it has zero topological entropy, and Theorem 1 applies. We then obtain a conjugate IET $T_{L_{t m}}$ with $B_{L_{t m}}$ as set of separation points. By Proposition 3.1.7(d) and since $\left\{a w_{2}, b w_{2}\right\} \subset \phi^{-1}(\iota(\bar{I}))$ by definition of $Z_{0}$, we have $T_{L_{t m}}\left(\phi_{\mu}\left(a w_{2}\right)\right)=\phi_{\mu}\left(\sigma\left(a w_{2}\right)\right)=\phi_{\mu}\left(w_{2}\right)=\phi_{\mu}\left(\sigma\left(b w_{2}\right)\right)=T_{L_{t m}}\left(\phi_{\mu}\left(b w_{2}\right)\right)$. Hence $T_{L_{t m}}$ is not injective.

Now, by Lemma 3.2.9, $\phi_{\mu}\left(a w_{2}\right)$ and $\phi_{\mu}\left(b w_{2}\right)$ must belong to the set of discontinuities $\mathcal{D}_{L_{t m}, \text { acc, } r}$. Note that this is coherent with the fact that by Lemma 3.2.5 $\left\{a w_{1}, b w_{1}, a w_{2}, b w_{2}\right\}=W_{\mathrm{SP}_{L_{t m}}}$, so that $\left\{\phi_{\mu}\left(a w_{2}\right), \phi_{\mu}\left(b w_{2}\right)\right\}=B_{\mathrm{SP}_{L_{t m}}}$, and that by definition, $B_{\mathrm{SP}_{L_{t m}}}=B_{L_{t m}, \text { acc }, r} \supseteq \mathcal{D}_{L_{t m}, \text { acc }, r}$.

Also, according to the construction behind Lemma 3.2.5, the set of separation points $B_{L}$ is always infinite while including the set $\mathcal{D}_{L}$ which can be in fact finite (see e.g. Corollary 3.3.4 further).

Example 3.2.12. (The set $\mathcal{D}_{L}$ of $T_{L}$ can be infinite) Let us consider again the ThueMorse associated shift $L_{t m}$ and its associated definitions. If there were only finitely many discontinuities for $T_{L_{t m}}$, we could assume there is an open interval $J \subset I$, having $\phi_{\mu}\left(a w_{2}\right)$ as the left endpoint, on which $T_{L_{t m}}$ is continuous. We could also assume $J$ avoids the finite set $\phi_{\mu}\left(W_{\mathrm{SP}_{L_{t m}}}\right)$, hence $J$ would be contained in a subset of $I$ where $T_{L_{t m}}$ is injective. But $T_{L_{t m}}\left(\phi_{\mu}\left(a w_{2}\right)\right)=T_{L_{t m}}\left(\phi_{\mu}\left(b w_{2}\right)\right)$, so by right-continuity, $T_{L_{t m}}$ cannot be injective on $J$ and there is no such interval.

3.3. Codings and reconstructions. In this section, we describe some more relationships between $L$ and $T_{L}$.

First, an IET $T$ is said to be minimal if all its orbits are dense in I. Next, a monotonicity partition $P$ of $I$ with respect to $T$ is a finite partition made of right-open intervals on each of which $T$ is increasing, but not necessarily continuous. Recall then that by Lemma 3.2.2, the conjugate IETs given by Theorem 1 are piecewise increasing, thus admitting monotonicity partitions. The associated coding of the orbits of $T$ with a partition $P$ is based on a map $\alpha$ assigning a distinct letter of an alphabet $A$ to each interval of $P$, so that

$$
\begin{aligned}
\operatorname{cod}_{0, P}: \quad I & \rightarrow A \\
x & \mapsto \alpha(J) \quad \text { if } x \in J, \text { with } J \in P,
\end{aligned}
$$

which is extended as

$$
\begin{aligned}
\operatorname{cod}_{P}: I & \rightarrow A^{\mathbb{N}} \\
x & \mapsto \operatorname{cod}_{0, P}(x) \operatorname{cod}_{0, P}(T(x)) \operatorname{cod}_{0, P}\left(T^{2}(x)\right) \ldots
\end{aligned}
$$


The word $\operatorname{cod}_{P}(x)$ is called the symbolic orbit of $x$ by $\operatorname{cod}_{P}$, and the closure $\overline{\operatorname{cod}_{P}(I)}$, denoted by $L_{P}$, is called the associated shift of $\operatorname{cod}_{P}$. A lexicographic order on $L_{P}$ is induced by the order over $A$ determined by the order of the component intervals of $P$ on $I$, that is, $\operatorname{cod}_{0, P}(x)<\operatorname{cod}_{0, P}\left(x^{\prime}\right)$, if $x \in J, x^{\prime} \in J^{\prime}$ with $J<J^{\prime}$, i.e. $J$ occurs before $J^{\prime}$ on $I$.

The next two technical lemmas make it possible to apply Theorem 1 to $L_{P}$.

LEMMA 3.3.1. Let $T$ be a piecewise increasing IET, and let $P$ be a monotonicity partition of $I$. Then $\operatorname{cod}_{P}$ is non-decreasing, Borel measurable, and right-continuous. Moreover, if $T$ is minimal, $\operatorname{cod}_{P}$ is increasing.

Proof. Let $x, x^{\prime} \in I$, with $x<x^{\prime}$. If $x \in J$ and $x^{\prime} \in J$, where $J, J^{\prime}$ are intervals of $P$ with $J<J^{\prime}$, we have $\operatorname{cod}_{0, P}(x)<\operatorname{cod}_{0, P}\left(x^{\prime}\right)$, hence $\operatorname{cod}_{P}(x)<\operatorname{cod}_{P}\left(x^{\prime}\right)$. Otherwise, $J=J^{\prime}$, so that $\operatorname{cod}_{0, P}(x)=\operatorname{cod}_{0, P}\left(x^{\prime}\right)$. Since on the intervals of $P$, we have $T(x)<T\left(x^{\prime}\right)$, we can inductively apply the same argument until for some $n>0, \operatorname{cod}_{0, P}\left(T^{n}(x)\right)<$ $\operatorname{cod}_{0, P}\left(T^{n}\left(x^{\prime}\right)\right)$, in which case $\operatorname{cod}_{P}(x)<\operatorname{cod}_{P}\left(x^{\prime}\right)$, and if there is no such $n$, then $\operatorname{cod}_{P}(x)=\operatorname{cod}_{P}\left(x^{\prime}\right)$. Hence, $\operatorname{cod}_{P}$ is non-decreasing. If $T$ is minimal, the above $n$ always exists since $T^{n}(x)$ can be arbitrarily close to the left end of an interval in $P$, and as long as both $T^{n}(x)$ and $T^{n}\left(x^{\prime}\right)$ are in the same interval of $P$, we have $T^{n}\left(x^{\prime}\right)-T^{n}(x) \geq x^{\prime}-x$. Hence, $\operatorname{cod}_{P}(x)<\operatorname{cod}_{P}\left(x^{\prime}\right)$.

The $\operatorname{map} \operatorname{cod}_{P}$ is the product of the $\operatorname{maps} \operatorname{cod}_{0, P} \circ T^{i}, i \geq 0$, each being continuous on the interiors of the intervals of a finite partition of $I$, since $T^{i}$ is piecewise increasing. Hence $\operatorname{cod}_{P}$ is Borel measurable. Likewise, each $\operatorname{cod}_{0, P} \circ T^{i}$ is right-continuous since $\operatorname{cod}_{P}$ and $T^{i}$ are right-continuous, hence $\operatorname{cod}_{P}$ is too.

From the above lemma, the image by $\operatorname{cod}_{P}$ of the Lebesgue measure on $I$ is a Borel measure on $L_{P}$ that we denote by $\mu_{P}$. Since $\operatorname{cod}_{P}$ conjugates $\sigma$ and $T, \mu_{P}$ is invariant by $\sigma$. Also, as $\operatorname{cod}_{P}$ is right-continuous, when $T$ is minimal, $L_{P}$ is aperiodic minimal, and $\mu_{P}$ is non-atomic and positive on the cylinder sets of $L_{P}$.

LEMMA 3.3.2. Let $T$ be a piecewise increasing and minimal IET. Let $P$ be a monotonicity partition of $I$, and let $L_{P}$ be the associated shift. Then $\mu_{P}\left(\operatorname{SP}_{L_{P}}\right)=0$.

Proof. By definition of $\mu_{P}$ for all $x \in I, \mu_{P}\left(\left[w_{L_{P}, \min }, \operatorname{cod}_{P}(x)\right]\right)$ is equal to the Lebesgue measure of $\operatorname{cod}_{P}^{-1}\left(\left[w_{L_{P}, \min }, \operatorname{cod}_{P}(x)\right)\right)$, that is, of $[0, x)$, since by Lemma 3.3.1 $\operatorname{cod}_{P}$ is increasing. Thus, using the map $\phi_{\mu_{P}}$ on $L_{P}$ for all $i \in I$,

$$
\phi_{\mu_{P}}\left(\operatorname{cod}_{P}(x)\right)=x .
$$

By Lemma 3.1.5 and by definition of $Z_{0}$, recall that if $x \notin Z_{0}, \phi_{\mu_{P}}^{-1}(x)$ is well-defined, and by equality (8) is equal to $\operatorname{cod}_{P}(x)$. If $x \in Z_{0}, \phi_{\mu_{P}}^{-1}(x)$ consists of two words, one of them being $\operatorname{cod}_{P}(x)$. Accordingly, $\operatorname{cod}_{P}(I)$ misses some words of $L_{P}$, but $L_{P} \backslash \operatorname{cod}_{P}(I)$ is countable, since $Z_{0}$ is countable.

Let us now estimate the measure of $\sigma^{-1}\left(\mathrm{SP}_{L_{P}}\right)$. We define the subset $W=$ $\sigma^{-1}\left(\operatorname{SP}_{L_{P}}\right) \cap\left(L_{P} \backslash \operatorname{cod}_{P}(I)\right)$, which is countable too. Let $w_{0} \in \sigma^{-1}\left(\mathrm{SP}_{L_{P}}\right)$. By definition of $\operatorname{SP}_{L_{P}},\left\{w_{0}\right\} \subsetneq \sigma^{-1}\left(\sigma\left(w_{0}\right)\right)$, and there are three cases.

- $w_{0} \in W$ : thus $w_{0} \in \sigma^{-1}(\sigma(W))$. 
- $w_{0} \notin W$ and there is $w_{1} \in \sigma^{-1}\left(\sigma\left(w_{0}\right)\right)$ such that $w_{1} \in W$. Thus $w_{0} \in \sigma^{-1}(\sigma(W))$ too, since $\sigma\left(w_{0}\right)=\sigma\left(w_{1}\right)$.

- $w_{0} \notin W$ and there is no $w_{1} \in \sigma^{-1}\left(\sigma\left(w_{0}\right)\right)$ such that $w_{1} \in W$. Thus there are $x_{0}, x_{1} \in I$ such that $\left\{w_{0}, w_{1}\right\} \subset \sigma^{-1}\left(\sigma\left(w_{0}\right)\right)$ with $w_{0}=\operatorname{cod}_{P}\left(x_{0}\right), w_{1}=\operatorname{cod}_{P}\left(x_{1}\right)$, and $x_{0} \neq x_{1}$ since $w_{0} \neq w_{1}$. By definition of $\operatorname{cod}_{P}$, for all $x \in I$,

$$
\operatorname{cod}_{P}(T(x))=\sigma\left(\operatorname{cod}_{P}(x)\right) .
$$

Since $\sigma\left(\operatorname{cod}_{P}\left(x_{0}\right)\right)=\sigma\left(\operatorname{cod}_{P}\left(x_{1}\right)\right)$, then $\operatorname{cod}_{P}\left(T\left(x_{0}\right)\right)=\operatorname{cod}_{P}\left(T\left(x_{1}\right)\right)$, that is, since $\operatorname{cod}_{P}$ is injective, $T\left(x_{0}\right)=T\left(x_{1}\right)$. Hence according to Definition $2, x_{0}, x_{1} \in \mathcal{D}_{\text {acc }, r}$, and $w_{0} \in \sigma^{-1}\left(\operatorname{cod}_{P}\left(\mathcal{D}_{\mathrm{acc}, r}\right)\right)$.

Thus $\sigma^{-1}\left(\operatorname{SP}_{L_{P}}\right) \subseteq \sigma^{-1}(\sigma(W)) \cup \sigma^{-1}\left(\operatorname{cod}_{P}\left(\mathcal{D}_{\text {acc }, r}\right)\right)$. Then, $\mu_{P}\left(\sigma^{-1}(\sigma(W))=0\right.$, since $\sigma^{-1}(\sigma(W))$ is countable and $\mu_{P}$ is non-atomic. Next, $\mu_{P}\left(\sigma^{-1}\left(\operatorname{cod}_{P}\left(\mathcal{D}_{\text {acc }, r}\right)\right)\right)=$ $\mu_{P}\left(\operatorname{cod}_{P}\left(\mathcal{D}_{\text {acc }, r}\right)\right)$ since $\mu_{P}$ is preserved by $\sigma$. Now, since $\mu_{P}$ is the image of the Lebesgue measure on $I$ by $\operatorname{cod}_{P}$ and $\mathcal{D}_{\text {acc }, r}$ has measure $0, T$ being an IET, $\mu_{P}\left(\operatorname{cod}_{P}\left(\mathcal{D}_{\text {acc }, r}\right)\right)=0$, so $\mu_{P}\left(\sigma^{-1}\left(\operatorname{cod}_{P}\left(\mathcal{D}_{\mathrm{acc}, r}\right)\right)\right)=0$ too. Hence $\mu_{P}\left(\sigma^{-1}\left(\operatorname{SP}_{L_{P}}\right)\right)=0$, and again by measure preservation, $\mu_{P}\left(\mathrm{SP}_{L_{P}}\right)=0$.

Then, given a monotonicity partition $P$, Lemma 3.2.5 applies to $L_{P}$ measured by $\mu_{P}$, so that Theorem 1 applies to $L_{P}$ too, and we can get a conjugate IET $T_{L_{P}}$.

Proposition 3.3.3. Let $T$ be a piecewise increasing and minimal IET. Let $P$ be a monotonicity partition of $I$, and let $L_{P}$ be the associated shift with its conjugate IET $T_{L_{P}}$. Then $T_{L_{P}}=T$, i.e.

$$
\text { for every monotonicity partition } P: T \stackrel{\operatorname{cod}_{P}}{\longrightarrow} L_{P} \stackrel{\text { Theorem } 1}{\longrightarrow} T_{L_{P}}=T .
$$

Proof. In equality (8), $T(x)$ can replace $x$, and by using (9), for all $x \in I$, we get

$$
T(x)=\phi_{\mu_{P}}\left(\operatorname{cod}_{P}(T(x))\right)=\phi_{\mu_{P}}\left(\sigma\left(\operatorname{cod}_{P}(x)\right)\right) .
$$

Also, right-continuity of $\operatorname{cod}_{P}$ (see Lemma 3.3.1) means that for every $x \in I, \operatorname{cod}_{P}(x)$ is the infimum of $\left\{\operatorname{cod}_{P}(y) \mid y>x, y \in I\right\}$. Thus $\operatorname{cod}_{P}(x)$ cannot be the right endpoint of a cylinder in $L_{P}$, hence $\operatorname{cod}_{P}(x) \in \phi^{-1}(\iota(I))$. In fact, since $\phi_{\mu_{P}}=\kappa \circ \phi$, and since $\iota \circ \kappa$ is the identity on $\phi\left(\operatorname{cod}_{P}(I)\right)$, we get $\operatorname{cod}_{P}(x)=\phi^{-1}(\iota(x))$ by composing equality (8) to the left with $\phi^{-1} \circ \iota$. By Proposition 3.1.7(d), $T_{L_{P}}\left(\phi_{\mu_{P}}(w)\right)=\phi_{\mu_{P}}(\sigma(w))$ on $\phi^{-1}(\iota(I))$, thus with $w=\phi^{-1}(\iota(x))$, for all $x \in I$,

$$
T_{L_{P}}(x)=\phi_{\mu_{P}}\left(\sigma\left(\operatorname{cod}_{P}(x)\right)\right),
$$

whence $T$ and $T_{L_{P}}$ coincide on $I$.

Of course the above result applies to minimal finite IETs, as these are piecewise increasing by definition. In this case, the partition $P$ can be the one induced by the set $\mathcal{D}$ of discontinuities, or by any finite $B \supseteq \mathcal{D}$. Then, $\operatorname{cod}_{P}$ becomes the usual natural coding of the orbits of $T$ with $B$ as set of separation points [Kea75], that is, a coding based on the assignment of a distinct letter to each component of a partition on which $T$ is continuous in the interior of these components. 
COROLLARY 3.3.4. Let $T$ be a finite minimal IET. Let $L$ be the associated shift given by a natural coding of $T$, with its conjugate IET $T_{L}$. Then $T=T_{L}$.

Note that in this finite IET case, $T$ is reobtained as $T_{L}$ from $L$, but according to the construction behind Lemma 3.2.5, the set of separation points $B_{L}$ is infinite, whereas $\mathcal{D} \subset$ $B_{L}$ is finite (see Example 5.2.4 (iv) further).

A shift $L$ can also be recovered from $T_{L}$. Recall that $P_{A}$ is the monotonicity partition of $I$ for $T_{L}$ relying on the letters of $A$ (see Lemma 3.2.2).

PROPOSITION 3.3.5. Let $L$ be any shift with a conjugate IET $T_{L}$ (Theorem 1 applies to $L$ ). Then for every $x \in I, \operatorname{cod}_{P_{A}}(x)=\phi^{-1}(\iota(x))$, so that $L_{P_{A}}=L$, i.e.

for the monotonicity partition $P_{A}: L \stackrel{\text { Theorem } 1}{\longrightarrow} T_{L_{P_{A}}} \stackrel{\operatorname{cod}_{P_{A}}}{\longrightarrow} L_{P_{A}}=L$.

Proof. For $x \in I$, by definition of $\operatorname{cod}_{0, P_{A}}$ and $P_{A}, \operatorname{cod}_{P_{A}}(x)=a$ when $x \in I_{L, a}$, since $\alpha\left(I_{L, a}\right)=a$. Next, by definition of $I_{L, a}, \phi^{-1}(\iota(x)) \in \operatorname{Cyl}(a)$, that is, the first letter of $\operatorname{cod}_{P_{A}}(x)$ and of $\phi^{-1}(\iota(x))$ are equal. To check equality on their second letters, that is, the first letter of $\sigma\left(\operatorname{cod}_{P_{A}}(x)\right)$ and of $\sigma\left(\phi^{-1}(\iota(x))\right)$, consider that since $x$ above can be arbitrary, the first letter of $\operatorname{cod}_{P_{A}}\left(T_{L}(x)\right)$ and of $\phi^{-1}\left(\iota\left(T_{L}(x)\right)\right)$ are equal too. Then by equality (9) $\operatorname{cod}_{P_{A}}\left(T_{L}(x)\right)=\sigma\left(\operatorname{cod}_{P_{A}}(x)\right)$, and by Proposition 3.1.7 (a,b) $\phi^{-1}\left(\iota\left(T_{L}(x)\right)\right)=\sigma\left(\phi^{-1}(\iota(x))\right)$. By inductively applying the same arguments, the equality holds for the next letters. Hence $\operatorname{cod}_{P_{A}}(I)=\phi^{-1}(\iota(I))$.

If an IET is given with an infinite set $B \supseteq \mathcal{D}$ of separation points, its natural coding is such that the coding alphabet must be then infinite too. However, in the case of $T_{L}$ with $B_{L}$ as set of separation points, this coding is related to $L$, that is, to a language over a finite alphabet. Indeed, let $A_{\infty}$ be an infinite alphabet and let $\alpha_{\infty}$ be a map assigning a distinct letter of $A_{\infty}$ to each component of the partition PART $_{L, I}$ (cf. p. 1946) so that its natural coding is defined as

$$
\begin{aligned}
\operatorname{cod}_{0, \infty}: \quad I & \rightarrow A_{\infty} \\
x & \mapsto \begin{cases}\alpha_{\infty}\left(I_{L}^{(k)}\right) & \text { if } x \in I_{L}^{(k)}, \\
\alpha_{\infty}(x) & \text { if } x \in B_{\mathrm{SP}_{L}} .\end{cases} \\
\operatorname{cod}_{\infty}: \quad I & \rightarrow A_{\infty}^{\mathbb{N}} \\
x & \mapsto \operatorname{cod}_{0, \infty}(x) \operatorname{cod}_{0, \infty}\left(T_{L}(x)\right) \operatorname{cod}_{0, \infty}\left(T_{L}^{2}(x)\right) \ldots
\end{aligned}
$$

Recall now that the partition $\mathrm{PART}_{L}$ of $L$ (cf. p. 1944) is made of the cylinders determined by the finite words $v^{(k)}$ in Fact $_{L}$ and by the infinite words in $W_{\mathrm{SP}_{L}}$. We consider then the following injective recoding map of the letters of $A_{\infty}$ :

$$
\begin{aligned}
\zeta_{\infty}: A_{\infty} & \rightarrow \text { Fact }_{L} \cup W_{\mathrm{SP}_{L}} \\
a & \mapsto \begin{cases}v^{(k)} & \text { if } a=\alpha_{\infty}\left(I_{L}^{(k)}\right), \\
w & \text { if } a=\alpha_{\infty}(x), \text { where } x \in B_{\mathrm{SP}_{L}} \text { and } \phi^{-1}(\iota(x))=w .\end{cases}
\end{aligned}
$$

Next, we define

$$
\delta_{0, \infty}: L \rightarrow A_{\infty}
$$

where for each $w \in L$ : 
- $\delta_{0, \infty}(w)=\left(\zeta_{\infty}\right)^{-1}(a u)$, if $a u$ occurs as a prefix of $w$, with $a \in A$, and $u \in$ Fact $_{L}$ is non-left special, that is, by the construction of $\operatorname{PART}_{L}$ in Lemma 3.2.5, au is a factor $v^{(k)}$ such that $\mathrm{Cyl}_{L}\left(v^{(k)}\right) \in \mathrm{PART}_{L}$;

- $\delta_{0, \infty}(w)=\left(\zeta_{\infty}\right)^{-1}(w)$, if $w$ is of the form $w=a w^{\prime}$, with $a \in A$, and $w^{\prime}$ is an infinite left special word in $\mathrm{SP}_{L}$, that is, $w \in W_{\mathrm{SP}_{L}}$.

The map $\delta_{0, \infty}$ is well-defined firstly because the two above cases cover $L$ and are independent, reflecting how PART $_{L}$ is built, and secondly because, if $a u$ exists, it is unique since the $v^{(k)}$ determining the cylinders of $\mathrm{PART}_{L}$ can never be prefixes to each other (these cylinders do not overlap). Then, $\delta_{0, \infty}$ is extended as follows:

$$
\begin{aligned}
\delta_{\infty}: L & \rightarrow A_{\infty}^{\mathbb{N}} \\
w & \mapsto \delta_{0, \infty}(w) \delta_{0, \infty}(\sigma(w)) \delta_{0, \infty}\left(\sigma^{2}(w)\right) \ldots
\end{aligned}
$$

We can now map the words in $L$ to symbolic orbits of $T_{L}$ obtained by $\operatorname{cod}_{\infty}$.

Proposition 3.3.6. Let $L$ be any shift with a conjugate IET $T_{L}$ (Theorem 1 applies to $L$ ). Then for every $x \in I, \operatorname{cod}_{\infty}(x)=\delta_{\infty}\left(\phi^{-1}(\iota(x))\right)$, i.e.

$$
L \stackrel{\text { Theorem } 1}{\longrightarrow} T_{L} \stackrel{\operatorname{cod}_{\infty}}{\longrightarrow} \operatorname{cod}_{\infty}(I)=\delta_{\infty}\left(\operatorname{cod}_{P_{A}}(I)\right) .
$$

Proof. For $x \in I$, the first letter of $\operatorname{cod}_{\infty}(x)$ and of $\delta_{\infty}(w)$, where $w=\phi^{-1}(\iota(x))$, are equal. Indeed, since $w$ belongs to some $\operatorname{Cyl}\left(v^{(k)}\right)$ or to $W_{\mathrm{SP}_{L}}, \delta_{0, \infty}(w)$ recovers the letter assigned by $\alpha_{\infty}$ to the component of $\mathrm{PART}_{L, I}$ containing $x$, i.e. $\operatorname{cod}_{\infty}(x)$. Next, $\quad \sigma\left(\operatorname{cod}_{\infty}(x)\right)=\operatorname{cod}_{\infty}\left(T_{L}(x)\right) \quad$ and $\sigma\left(\delta_{\infty}\left(\phi^{-1}(\iota(x))\right)\right)=\delta_{\infty}\left(\sigma\left(\phi^{-1}(\iota(x))\right)\right)=$ $\delta_{\infty}\left(\phi^{-1}\left(\iota\left(T_{L}(x)\right)\right)\right)$ so that the second letters are also equal. By inductively applying the same arguments, we obtain $\operatorname{cod}_{\infty}(x)=\delta_{\infty}\left(\phi^{-1}(\iota(x))\right)$. Hence $\operatorname{cod}_{\infty}(I)=$ $\delta_{\infty}\left(\phi^{-1}(\iota(I))\right)$, and by Proposition 3.3.5, $\delta_{\infty}\left(\phi^{-1}(\iota(I))\right)=\delta_{\infty}\left(\operatorname{cod}_{P_{A}}(I)\right)$.

This mapping of $L$ to the natural coding of $T_{L}$ will be in use in the next section.

\section{The linear complexity shift case}

We now focus on shifts $L$ with linear complexity, i.e. those such that $p_{L}(n)=O(n)$, and prove Theorem 2. For instance, aperiodic minimal shifts associated with primitive substitutions have linear complexity [Pan84, Qué10], e.g. the shift associated with the Thue-Morse substitution. Since these shifts form a specific family of shifts with zero topological entropy, the idea behind Theorem 2 is to investigate the properties of their conjugate IETs as given by Theorem 1 .

4.1. Almost finite interval exchange transformations. We begin by defining a specific kind of IETs.

Definition 3. An IET $T$ with a set $\mathcal{D}$ of discontinuities is almost finite if:

(1) $T$ is piecewise increasing on $I$;

(2) the set $\mathcal{D}_{\text {acc }}$ of accumulation points of $\mathcal{D}$ is finite;

(3) the points in $\mathcal{D}$ belong to a finite number of full orbits of $T \dagger$.

$\dagger$ A sufficient condition for minimality of a finite IET is that the full orbits of the discontinuity points are infinite and distinct [Kea75] (cf. the infinite distinct orbit condition (IDOC)). An almost finite IET with an infinite number of discontinuities is thus far from satisfying the IDOC. 
In the above definition-like in Definitions 1 and $2-\mathcal{D}$ can be replaced by a nullmeasure set of separation points $B$ such that $B \supset \mathcal{D}$. Note also that a finite IET is almost finite. Now, the first property of almost finiteness is always satisfied by a conjugate IET $T_{L}$ (see Lemma 3.2.2). About the second property, when $L$ has linear complexity, we first show the following technical result.

LEMMA 4.1.1. Let L be a minimal shift with linear complexity. Then $\mathrm{SP}_{L}$ is finite.

Proof. For each finite $r \leq\left|\mathrm{SP}_{L}\right|$, there is $n_{0}>0$ such that for all $n \geq n_{0}$, the number of distinct length- $n$ prefixes of the words in $\mathrm{SP}_{L}$ is at least $r$. If $\operatorname{sp}_{L, l}(n)$ denotes the number of left special factors in $\operatorname{Fact}_{L}(n)$, then $\operatorname{sp}_{L, l}(n) \geq r$. Since $L$ is minimal, Fact $_{L}$ is prolongable, and thus for all $n \geq 1, p_{L}(n+1)-p_{L}(n) \geq \operatorname{sp}_{L, l}(n)$. Therefore, if $\mathrm{SP}_{L}$ was infinite, for every $r \in \mathbb{N}$, there would exist $n_{0}>0$ such that for all $n \geq n_{0}$, $p_{L}(n+1)-p_{L}(n) \geq r$, whence $p_{L}(n) \neq O(n)$.

Then we have the following.

LEMMA 4.1.2. Let $L$ be an aperiodic minimal shift with linear complexity, and let $T_{L}$ be its conjugate IET. Then the set $\mathcal{D}_{L \text {, acc }}$ of the accumulation points of the set $\mathcal{D}_{L}$ of the discontinuities of $T_{L}$ is finite.

Proof. By definition, $\mathcal{D}_{L} \subseteq B_{L}$. Next, $B_{L \text {, acc }}$ is determined by $W_{\mathrm{SP}_{L}}$ since $\phi_{\mu}\left(W_{\mathrm{SP}_{L}}\right)=$ $B_{L, \text { acc }}$, and we also have $W_{\mathrm{SP}_{L}}=\sigma^{-1}\left(\mathrm{SP}_{L}\right)$. The alphabet $A$ is finite, and by Lemma 4.1.1 $\mathrm{SP}_{L}$ is too, whence the result.

In order to prove the third property of almost finiteness for $T_{L}$, the idea is to study similar relationships to known ones for finite IETs, between natural codings, complexity of the associated shifts, and distinctiveness of the orbits of the separation points [Kea75]. However, as seen in $\S 3.3$, a natural coding $\operatorname{cod}_{\infty}$ of an infinite IET $T$ uses an infinite alphabet $A_{\infty}$. To deal with this situation, we shall code the orbits of $T$ using finer and finer finite partitions of $I$, together with finite alphabets with more and more letters, as is developed in the next section.

4.2. A finite number of full orbits. For an IET $T$ with $B \supset \mathcal{D}$ as set of separation points, let $B_{\text {acc }}$ denote the set of accumulation points of $B$ in $\bar{I}$, and let $B_{\text {acc }}=B_{\text {acc }}^{1} \sqcup B_{\text {acc }}^{2}$, where $B_{\text {acc }}^{1}$ denotes the one-sided accumulation points, and $B_{\text {acc }}^{2}$ the two-sided ones. An ordered finite subset $B_{m}=\left\{0=b_{0}, b_{1}, \ldots, b_{m-1}\right\}, m>0$, of $B$ is said to be admissible if for $B_{m} \cup\left\{b_{m}=1\right\}$, each induced interval $I_{m, i}=\left[b_{i}, b_{i+1}\right)$ has one of the following types.

(1) $I_{m, i}$ is an interval of $T$ with respect to $B\left(b_{i}\right.$ is the only point of $B$ in $\left.I_{m, i}\right)$.

(2) $I_{m, i}$ covers infinitely many consecutive intervals of $T$, and either:

(a) $\left[b_{i}, b_{i+1}\right]$ has exactly one point of $B_{\text {acc }}$, belonging to $B_{\text {acc }}^{1}$, either $b_{i}$ or $b_{i+1}$;

(b) $\left(b_{i}, b_{i+1}\right)$ has exactly one point of $B_{\text {acc }}$, belonging to $B_{\text {acc }}^{2}$, with $b_{i}, b_{i+1} \notin B_{\text {acc }}$.

Thus, an admissible $B_{m}$ induces a finite partition $\bigsqcup_{i} I_{m, i}$ of $I$, where for each point $x \in B_{L, \text { acc }}$, there is exactly one $I_{m, i_{x}}$ such that almost every point of any sequence of points of $B$ converging to $x$ lies in $I_{m, i_{x}}$. For instance, here is a possible subset $B_{4}$ of a set $B$ with two accumulation points $x_{1} \in B_{\text {acc }}^{1}$ and $x_{2} \in B_{\text {acc }}^{2}$, such that the induced intervals $I_{4,0}, I_{4,2}$ are of type $1, I_{4,1}$ is of type $2 \mathrm{a}$, and $I_{4,3}$ is of type $2 \mathrm{~b}$. 


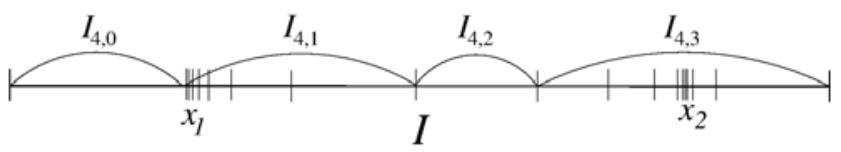

LEMMA 4.2.1. Let $B$ be a set of separation points. Then $B_{\text {acc }}$ is finite if and only if for some $m>0$, there is an admissible $B_{m} \subset B$.

Proof. $(\Leftarrow)$ Trivial. $(\Rightarrow)$ First, for each point $x \in B_{\text {acc }}^{2}$ there is an interval of type $2 \mathrm{~b}$ containing it, because such an $x$ is the intersection of a sequence of closed nested intervals with endpoints in $B$, and if every interval in the sequence contained a point in $B_{\text {acc }}$, then $B_{\text {acc }}$ would be infinite. Next, by the same argument, each $x \in B_{\text {acc }}^{1}$, which is the intersection of a sequence of nested closed intervals to its right, is the left endpoint of an interval of type $2 \mathrm{a}$, while in its other side, $x$ is the right endpoint of an interval of type 1 , or else $x=0$. The same occurs the other way around when the accumulation is on the left. Then there are finitely many points of $B$ in the complement of the union of the above intervals and together with the endpoints of those intervals, they form an admissible $B_{m}$.

Given an admissible set $B_{m}$, let $A_{m}$ be an alphabet with $m$ letters, and let $\alpha_{m}$ be a map assigning a distinct letter in $A_{m}$ to each $I_{m, i}$ induced by $B_{m}$, so that

$$
\begin{aligned}
& \operatorname{cod}_{0, m}: \quad I \quad \rightarrow A_{m} \\
& x \mapsto \alpha_{m}\left(I_{m, i}\right), \quad \text { if } x \in I_{m, i} . \\
\operatorname{cod}_{m}: \quad & \rightarrow A_{m}^{\mathbb{N}} \\
x & \mapsto \operatorname{cod}_{0, m}(x) \operatorname{cod}_{0, m}(T(x)) \operatorname{cod}_{0, m}\left(T^{2}(x)\right) \ldots
\end{aligned}
$$

The associated shift $L_{m}$ of $\operatorname{cod}_{m}$ is then defined as $\overline{\operatorname{cod}_{m}(I)}$, as usual.

Now, let $L$ be a shift over $A$, with a conjugate IET $T_{L}$ having $B_{L}$ as set of separation points, and $B_{L \text {, acc }}=B_{L, \text { acc }}^{1} \sqcup B_{L \text {,acc }}^{2}$ as accumulation point sets. Let $B_{L, m}$ be an admissible subset of $B_{L}$, inducing a finite partition $\bigsqcup_{i} I_{L, m, i}$ of $I$. Then, we define a recoding map similar to and based on $\zeta_{\infty}$ (see p. 1952), sending each letter of $A_{m}$ to words over $A$, which are either $v^{(k)}$ or common prefixes of sets of $v^{(k)}$ (the $v^{(k)}$ determining the cylinder components of $\mathrm{PART}_{L}$ ):

$$
\zeta_{m}: A_{m} \rightarrow \text { Fact }_{L} \cup\left(\text { Fact }_{L} \times \text { Fact }_{L}\right),
$$

where for each $a \in A_{m}$ :

(1) $\zeta_{m}(a)=\zeta_{\infty}\left(\alpha_{\infty}\left(I_{L, m, i}\right)\right)$, if $a=\alpha_{m}\left(I_{L, m, i}\right)$ where $I_{L, m, i}$ is of type 1 , that is, $\zeta_{m}$ yields the same $v^{(k)}$ as $\zeta_{\infty}$ if $a$ is assigned to a type 1 interval;

(2) (a) $\zeta_{m}(a)=v_{\text {pref }}$, if $a=\alpha_{m}\left(I_{L, m, i}\right)$ where $I_{L, m, i}$ is of type 2a, and $v_{\text {pref }}$ is the longest common prefix of $\left\{\zeta_{\infty}\left(\alpha_{\infty}(J)\right) \mid J \in \mathrm{PART}_{L, I}, J \subset I_{L, m, i}\right\}$, that is, $\zeta_{m}$ yields the longest common prefix of the $v^{(k)}$ given by $\zeta_{\infty}$ applied to all the intervals covered by $I_{L, m, i}$;

(b) $\zeta_{m}(a)=\left(v_{\text {pref }, l}, v_{\text {pref }, r}\right)$, if $a=\alpha_{m}\left(I_{L, m, i}\right)$ where $I_{L, m, i}$ of type $2 \mathrm{~b}$, and $v_{\text {pref }, l}, v_{\text {pref }, r}$ are defined as follows: let $I_{L, m, i}=\left[b_{i}, b_{i+1}\right)$ be divided into $J_{l}=$ $\left[b_{i}, x\right)$ and $J_{r}=\left[x, b_{i+1}\right)$, where $x \in B_{L, \text { acc }}^{2}$ is the accumulation point in $I_{L, m, i}$; then $v_{\text {pref }, l}$ (respectively $\left.v_{\text {pref, } r}\right)$ is the longest common prefix of $\left\{\zeta_{\infty}\left(\alpha_{\infty}(J)\right) \mid\right.$ $\left.J \in \operatorname{PART}_{L, I}, J \in J_{l}\right\}$ (respectively $J \in J_{r}$ ). 
The case 2(b) above deals with the possible existence of points $x \in B_{L \text {,acc }}^{2}$ such that $\phi_{\mu}^{-1}(x)$ is made of two consecutive distinct words in $W_{\mathrm{SP}_{L}}$.

We show then that if $m$ is sufficiently large, the map $\zeta_{m}$ can be used like $\zeta_{\infty}$ to define a map $\delta_{m}$ similar to $\delta_{\infty}$. First of all, we say a sequence $\left\{B_{m}\right\}_{m \geq m_{0}}$, with $m_{0}>0$, is admissible if for all $m \geq m_{0}$ : (i) $B_{m}$ is an admissible subset of $B$; (ii) $\left|B_{m+1}\right|=\left|B_{m}\right|+1$; (iii) $B=$ $\bigcup_{m \geq m_{0}} B_{m} \cup\left(B_{\text {acc }} \backslash\{1\}\right)$.

LEMMA 4.2.2. Let $B$ be a set of separation points. Then $B_{\mathrm{acc}}$ is finite if and only if for some $m_{0}>0$, there is an admissible sequence $\left\{B_{m}\right\}_{m \geq m_{0}}$.

Proof. $(\Leftarrow)$ A consequence of Lemma 4.2.1. $(\Rightarrow)$ By Lemma 4.2.1 also, for some $m_{0}>0$ there is an admissible $B_{m_{0}}$ of $B$. If the first point $x$ of $B_{\text {acc }}$ in $I$ lies in an interval $I_{m_{0}, i}$ of type $2 \mathrm{~b}$, we add to $B_{m_{0}}$ the smallest point of $B$ in the interior of $I_{m_{0}, i}$ to obtain $B_{m_{0}+1}$, then the greatest one to obtain $B_{m_{0}+2}$. If $x$ lies in $I_{m_{0}, i}$ of type $2 \mathrm{a}$ we do the same, but using the smallest or the greatest point of $B$ in the interior of $I_{m_{0}, i}$, according to whether the point is an accumulation point on the left or the right. Then we proceed to the next point of $B_{\text {acc }}$, and so on, going back to the first one after having processed the last one.

We denote by $F_{m, 1}$ (respectively $F_{m, 2}$ ) the set of the $v^{(k)}$ (respectively the prefixes of the $\left.v^{(k)}\right)$ given by $\zeta_{m}\left(\alpha_{m}\left(I_{L, m, i}\right)\right)$ when $I_{L, m, i}$ is of type 1 (respectively type 2, including $v_{\text {pref }, l}$ and $v_{\text {pref,r } r}$ as distinct words when $I_{L, m, i}$ is of type $2 \mathrm{~b}$ ). The technicalities in the proof of the following lemma come from the topological differences between $L$ and $I$.

LEMMA 4.2.3. Let $L$ be a shift with a conjugate $T_{L}$ and an admissible sequence $\left\{B_{L, m}\right\}_{m \geq m_{0}}, m_{0}>0$. Then there is $m_{1} \geq m_{0}$, such that for all $m \geq m_{1}$, the map $\zeta_{m}$ is injective, such that for $i=1,2, F_{m, i}$ does not include the empty word, nor words prefix to each other, and no word in $F_{m, 1}$ is a prefix of a word in $F_{m, 2}$.

Proof. By the construction of $\mathrm{PART}_{L}$ in Lemma 3.2.5, the set $F_{m, 1}$, being made of $v^{(k)}$, has the first two required properties. Next, a word $v$ in $F_{m, 1}$ cannot be a prefix of any word in $F_{m, 2}$, since $v$ would be then a common prefix of words of type $v^{(k)}$, and again the $v^{(k)}$ are not prefixes to each other.

Now, from the point-of-view of $L$, each word $w \in W_{\mathrm{SP}_{L}}$ is an accumulation of endpoints of cylinder components of $\mathrm{PART}_{L}$, and can be seen as the intersection of a sequence of nested cylinders of $L$ (see again the proof of Lemma 3.2.5). Also, by Lemma 4.2.2, the set $B_{L \text {, acc }}$ must be finite, so that $W_{\mathrm{SP}_{L}}$ is too. Therefore, there exists $n>0$ such that at the $n$th step of the construction of $\mathrm{PART}_{L}$, the nested cylinders to be refined at each further step are such that they contain only one word in $W_{\mathrm{SP}_{L}}$, and such that they are all disjoint. Thus for each $w \in W_{\mathrm{SP}_{L}}$, there is a sequence of nested cylinders $\left\{C_{w, j}\right\}_{j \in \mathbb{N}^{*}}$ such that $\bigcap_{j \in \mathbb{N}^{*}} C_{w, j}=\{w\}$, disjoint from the other sequences, and having one of these two forms: $\left(\mathrm{a}_{L}\right)\left\{C_{w, j}\right\}_{j \in \mathbb{N}^{*}}$ is such that $w$ is the smallest or the greatest element of every $C_{w, j}$, so that $w$ is a one-sided accumulation word in $L$;

$\left(\mathrm{b}_{L}\right)\left\{C_{w, j}\right\}_{j \in \mathbb{N}^{*}}$ is such that $w$ belongs to the interior of every cylinder $C_{w, j}$, so that $w$ is a two-sided accumulation word in $L$. 
From the point-of-view of $I$, the corresponding sequences $\left\{\phi_{\mu}\left(C_{w, j}\right)\right\}_{j \in \mathbb{N}^{*}}$ are sequences of nested closed intervals with pairwise disjoint interiors, such that $\bigcap_{j \in \mathbb{N}^{*}} \phi_{\mu}\left(C_{w, j}\right)=\left\{\phi_{\mu}(w)\right\}$, with $\phi_{\mu}(w) \in B_{L, \text { acc }}$, taking one of these two forms:

(a $I) \quad\left\{\phi_{\mu}\left(C_{w, j}\right)\right\}_{j \in \mathbb{N}^{*}}$ is such that $\phi_{\mu}(w)$ is equal to the right or the left endpoint of every interval $\phi_{\mu}\left(C_{w, j}\right)$ so that $\phi_{\mu}(w) \in B_{L, \text { acc }}^{1}$ or $B_{L, \text { acc }}^{2}$;

(b $\left.\mathrm{b}_{I}\right)\left\{\phi_{\mu}\left(C_{w, j}\right)\right\}_{j \in \mathbb{N}^{*}}$ is such that $\phi_{\mu}(w)$ belongs to the interior of $\phi_{\mu}\left(C_{w, j}\right)$, so that $\phi_{\mu}(w) \in B_{L, \text { acc }}^{2}$.

From the point-of-view of $\left\{B_{L, m}\right\}_{m \geq m_{0}}$, for each $m \geq m_{0}$, every $x \in B_{L, \text { acc }}$ is such that there is a $I_{L, m, i}$ of type 2 containing almost every point of any sequence in $B_{L}$ converging to $x$, so that putting $J_{x, m}=\overline{I_{L, m, i}}$, we get a sequence of nested intervals $\left\{J_{x, m}\right\}_{m \geq m_{0}}$, with $\bigcap_{m \geq m_{0}} J_{x, m}=\{x\}$, taking one of these three forms:

$\left(\mathrm{a}_{B}\right)\left\{J_{x, m}\right\}_{m \geq m_{0}}$ is made of type $2 \mathrm{a}$ intervals, i.e. $x \in B_{L, \text { acc }}^{1}$, where $\phi_{\mu}^{-1}(x)=w$, $w \in W_{\mathrm{SP}_{L}}$. There is then a corresponding sequence $\left\{C_{w, j}\right\}_{j \in \mathbb{N}^{*}}$ of form $\mathrm{a}_{L}$ with intersection $\{w\}$, and $\left\{\phi_{\mu}\left(C_{w, j}\right)\right\}_{j \in \mathbb{N}^{*}}$ of form a $\mathrm{a}_{I}$ with intersection $\{x\}$;

$\left(\mathrm{b}_{B}\right)\left\{J_{x, m}\right\}_{m \geq m_{0}}$ is made of type $2 \mathrm{~b}$ intervals, i.e. $x \in B_{L \text {, acc }}^{2}$, where either:

(i) $\phi_{\mu}^{-1}(x)=w$, with $w \in W_{\mathrm{SP}_{L}}$. There is then a corresponding sequence $\left\{C_{w, j}\right\}_{j \in \mathbb{N}^{*}}$ of form $\mathrm{b}_{L}$ with intersection $\{w\}$, and $\left\{\phi_{\mu}\left(C_{w, j}\right)\right\}_{j \in \mathbb{N}^{*}}$ of form $\mathrm{b}_{I}$ with intersection $\{x\}$;

(ii) $\phi_{\mu}^{-1}(x)=\left\{w, w^{\prime}\right\}$, with $w, w^{\prime} \in W_{\mathrm{SP}_{L}}$. There are then corresponding sequences $\left\{C_{w, j}\right\}_{j \in \mathbb{N}^{*}},\left\{C_{w^{\prime}, j}\right\}_{j \in \mathbb{N}^{*}}$ of form $a_{L}$, respectively with intersection $\{w\}$ and $\left\{w^{\prime}\right\}$, and $\left\{\phi_{\mu}\left(C_{w, j}\right)\right\}_{j \in \mathbb{N}^{*}},\left\{\phi_{\mu}\left(C_{w^{\prime}, j}\right)\right\}_{j \in \mathbb{N}^{*}}$ of form $\mathrm{a}_{I}$, both with intersection $\{x\}$.

Now, since the sequences $\left\{\phi_{\mu}\left(C_{w, j}\right)\right\}_{j \in \mathbb{N}^{*}}$ and $\left\{J_{x, m}\right\}_{m \geq m_{0}}$ have the same intersection point in $B_{L \text {, acc }}$, and because of the properties of the $C_{w, 1}$, there exists $m_{1}>0$, such that one of the following cases occur.

- $\left\{J_{x, m}\right\}_{m \geq m_{0}}$ is of form $\mathrm{a}_{B}$ or $\mathrm{b}_{B} \mathrm{i}$, and for all $m>m_{1}, J_{x, m} \subset \phi_{\mu}\left(C_{w, 1}\right)$, that is, the word determining the cylinder $C_{w, 1}$ is a common prefix of the words in $\left\{\zeta_{\infty}\left(\alpha_{\infty}\left(J_{x, m}\right)\right) \mid m>\right.$ $\left.m_{1}\right\}$, i.e. a prefix of the corresponding $v_{\text {pref }} \in F_{m, 2}$.

- $\left\{J_{x, m}\right\}_{m \geq m_{0}}$ is of form $\mathrm{b}_{B} \mathrm{ii}$, and for all $m>m_{1}, J_{x, m} \subset \phi_{\mu}\left(C_{w, 1}\right) \cup \phi_{\mu}\left(C_{w^{\prime}, 1}\right)$, that is, the two words determining $C_{w, 1}$ and $C_{w^{\prime}, 1}$ are respective common prefixes of the factors in $\left\{\zeta_{\infty}\left(\alpha_{\infty}\left(J_{l}\right)\right) \mid m>m_{1}\right\}$ and $\left.\zeta_{\infty}\left(\alpha_{\infty}\left(J_{r}\right)\right) \mid m>m_{1}\right\}$, where $J_{l}$ and $J_{r}$ are the two components of $J_{x, m} \backslash x$, i.e. prefixes of the corresponding $\left(v_{\text {pref }, l}, v_{\text {pref }, r}\right)$, with $v_{\text {pref }, l}, v_{\text {pref }, r} \in F_{m, 2}$.

Thus, for all $m \geq m_{1}$, since all the $C_{w, 1}$ are disjoint, the set $F_{m, 2}$ does not contain the empty word, nor words which are prefix to each other.

Similarly to $\delta_{0, \infty}$, we can now define $\delta_{0, m}$ for each $m>m_{1}$, using the properties of $\zeta_{m}$, so as to obtain letters of $A_{m}$ out of the prefixes of the words in $L$ :

$$
\delta_{0, m}: L \rightarrow A_{m}
$$

where for each $w \in L$ :

(1) $\delta_{0, m}(w)=\left(\zeta_{m}\right)^{-1}(a u)$, if $a u$ occurs as a prefix of $w$ in $F_{m, 1}$, that is, $a u$ is a factor $v^{(k)}$ which belongs to $\zeta_{m}\left(A_{m}\right)$, and such that $\mathrm{Cyl}_{L}\left(v^{(k)}\right) \in \operatorname{PART}_{L}$;

(2) or, one of the two following cases occurs:

- $\delta_{0, m}(w)=\left(\zeta_{m}\right)^{-1}\left(v_{\text {pref }}\right)$, if $v_{\text {pref }}$ occurs as a prefix of $w$ in $F_{m, 2}$, which belongs as a single word to $\zeta_{m}\left(A_{m}\right)$; 
- $\delta_{0, m}(w)=\left(\zeta_{m}\right)^{-1}\left(v_{\text {pref }, l}, v_{\text {pref }, r}\right)$, if $v_{\text {pref }, l}$ or $v_{\text {pref }, r}$ occurs as a prefix of $w$ in $F_{m, 2}$, which belongs to a pair $\left(v_{\text {pref }, l}, v_{\text {pref }, r}\right)$ in $\zeta_{m}\left(A_{m}\right)$.

This map is well-defined because, firstly, all the above cases cover $L$ by definition of $F_{m, 1}$ and $F_{m, 2}$, and secondly, the properties of $F_{m, 1}$ and $F_{m, 2}$ proved by Lemma 4.2.3 imply that taken in order, only one case applies for each $w \in L$. Accordingly, $\delta_{0, m}$ is extended as follows to obtain words in $A_{m}^{\mathbb{N}}$ from words in $L$ :

$$
\begin{aligned}
\delta_{m}: L & \rightarrow A_{m}^{\mathbb{N}} \\
w & \mapsto \delta_{0, m}(w) \delta_{0, m}(\sigma(w)) \delta_{0, m}\left(\sigma^{2}(w)\right) \ldots
\end{aligned}
$$

We then have a similar result to Proposition 3.3.6 ( $m_{1}$ comes from Lemma 4.2.3).

LEMMA 4.2.4. Let $L$ be any shift with a conjugate $T_{L}$ and an admissible sequence $\left\{B_{L, m}\right\}_{m \geq m_{1}}$. Then, for all $m \geq m_{1}$ and for all $x \in I, \operatorname{cod}_{m}(x)=\delta_{m}\left(\phi^{-1}(\iota(x))\right)$, i.e.

$$
L \stackrel{\text { Theorem } 1}{\longrightarrow} T_{L} \stackrel{\operatorname{cod}_{m}}{\longrightarrow} L_{m}=\delta_{m}(L) .
$$

Proof. For $x \in I$, the first letter of $\operatorname{cod}_{m}(x)$ and of $\delta_{m}(w)$, where $w=\phi^{-1}(\iota(x))$, are equal. Indeed, $w$ belongs to some cylinder determined by a word in $F_{m, 1} \cup F_{m, 2}$. Then, Case (1) of the $\delta_{0, m}(w)$ definition applies if and only if $x$ belongs to a type 1 interval and $\delta_{0, m}(w)$ recovers the letter assigned by $\alpha_{m}$ to this interval, i.e. $\operatorname{cod}_{m}(x)$. Otherwise, $x$ belongs to a type 2 interval, and Case (2) of the $\delta_{0, m}(w)$ definition necessarily applies, with the same effect. This is well-defined since by Lemma 4.2 .3 only words in $F_{m, 2}$ can be prefixes of words in $F_{m, 1}$. Next, similarly to Proposition 3.3.6, using the conjugacies given by Proposition 3.1.7, the same is true for the other letters of $\operatorname{cod}_{m}(x)$ and $\delta_{m}(w)$. Hence $\operatorname{cod}_{m}(I)=\delta_{m}\left(\phi^{-1}(\iota(I))\right)$.

LEMMA 4.2.5. Let $L$ be any shift with a conjugate $T_{L}$ and an admissible sequence $\left\{B_{L, m}\right\}_{m \geq m_{1}}$. Then, for all $m \geq m_{1}$, there exists $h_{m}>0$ such that for all $n>0, p_{L_{m}}(n) \leq$ $p_{L}\left(n+h_{m}\right)$,

Proof. Fix some $m \geq m_{1}$, and let $w \in L$ and $w^{\prime}=\delta_{m}(w) \in L_{m}$. Let $h_{m}$ be the maximum length of the factors in $F_{m, 1} \cup F_{m, 2}$. According to the definition of $\delta_{0, m}$ and to Lemma 4.2.4, for each $n>0$, the length- $\left(n+h_{m}\right)$ prefix of $w$ determines by $\delta_{m}$ at least one length- $n$ prefix of $w^{\prime}$, hence, $p_{w^{\prime}}(n) \leq p_{w}\left(n+h_{m}\right)$. Now, $L$ being minimal, $L_{m}$ is too, and $w, w^{\prime}$ are minimal words. Thus each of the factors in Fact $_{w}$, i.e. Fact Faccurs as a prefix in the words in $\left\{\sigma^{i}(w)\right\}_{i \in \mathbb{N}}$, and these factors determine Fact $w_{w^{\prime}}$, i.e. Fact $_{L_{m}}$. Hence for all $n>0, p_{L_{m}}(n) \leq p_{L}\left(n+h_{m}\right)$.

LEMMA 4.2.6. Let $L$ be an aperiodic minimal shift with linear complexity. Let $T_{L}$ be its conjugate IET. Then $B_{L}$ belongs to a finite number of full orbits of $T_{L}$.

Proof. According to the proof of Lemma 4.1.2, $B_{L \text {, acc }}$ is finite, thus according to Lemma 4.2.2 there is an admissible sequence $\left\{B_{L, m}\right\}_{m \geq m_{1}}$ of $B_{L}$, with $m_{1}>0$ given by Lemma 4.2.3. Fix some $m \geq m_{1}$ and consider the shift $L_{m}$ associated with $\operatorname{cod}_{m}$. For each $v \in$ Fact $_{L_{m}}$, let $E_{v}=\left\{x \in I \mid \operatorname{cod}_{0, m}(x) \operatorname{cod}_{0, m}\left(T_{L}(x)\right) \ldots \operatorname{cod}_{0, m}\left(T_{L}^{|v|-1}(x)\right)=v\right\}$, i.e. the set of the points in $I$ having symbolic orbits by $\operatorname{cod}_{m}$ with $v$ as the prefix (note that $E_{v}$ is not necessarily connected). For each $n>0, \bigsqcup_{v \in \operatorname{Fact}_{L_{m}}(n)} E_{v}$ is a partition of $I$, and the set 
$\left\{T_{L}^{-j}\left(B_{L, m}\right) \mid j=0, \ldots, n-1\right\}$ defines the endpoints of the interval components of this partition. Then, for any point $x \in B_{L, m}$, if the set $T_{L}^{-n}(x)$ has a point $y$ in the interior of some $E_{v}$ with $v \in \operatorname{Fact}_{L_{m}}(n)$, it means that to each side of $y$ there are components of $E_{v_{1}}$ and $E_{v_{2}}$, with $v_{1}, v_{2} \in \operatorname{Fact}_{L_{m}}(n+1)$ with $v$ as the prefix, and $v_{1} \neq v_{2}$. Thus $v$ is a right special factor and it induces an increase in the complexity $p_{L_{m}}$ of $L_{m}$.

Now, let $k_{m} \leq\left|B_{L, m}\right|=m$ be the number of distinct full orbits of $B_{L, m}$. Since $L_{m}$ is aperiodic minimal, each of these orbits may contain at most one occurrence of each point in $B_{L, m}$. And since $B_{L, m}$ is finite, in each of these orbits there is at least $-j \in \mathbb{Z}^{-}$for which a point of $B_{L, m}$ occurs in $\left\{T_{L}^{-j}\left(B_{L, m}\right) \mid j=0, \ldots\right\}$. Thus there are at least $k_{m}$ points in $B_{L, m}$ such that for all $n>0$, their preimages by $T_{L}^{-n}$ avoid $B_{L, m}$, and being in the interior of some $E_{v}$, they induce an increase of the number of the length- $n$ factors. Hence, for all $m \geq m_{1}$ and for all $n>0, k_{m} n \leq p_{L_{m}}(n)$. By Lemma 4.2.5, and since $L$ has linear complexity, there exists $a>1$ such that

$$
p_{L_{m}}(n) \leq p_{L}\left(n+h_{m}\right) \leq a\left(n+h_{m}\right),
$$

that is,

$$
k_{m} \leq a+\frac{a h_{m}}{n} .
$$

Since $n$ is a free variable, we have $k_{m} \leq a$ for all $m \geq m_{1}$, and thus letting $m$ go to infinity, this inequality holds also for $B_{L}$ of $T_{L}$.

We can then prove Theorem 2 as stated in the introduction.

Proof. Since $L$ has linear complexity, Theorem 1 applies, and we get a conjugate IET $T_{L}$. By Lemmas 3.2.2 and 4.1.2, $T_{L}$ has the two first properties of almost finiteness. Next, applying Lemma 4.2.6, and using the fact that $\mathcal{D}_{L} \subseteq B_{L}$, then $T_{L}$ has also the third property of almost finiteness.

\section{Building infinite interval exchange transformations}

In this section, we present results in the context of zero entropy about how to build effective approximations of the conjugate IETs $T_{L}$ given by Theorem 1 for a set of corresponding $\sigma$-invariant measures on the shifts $L$.

5.1. An approximation scheme. Given a shift $L$ over an ordered alphabet $A$, and its complexity $p_{L}$, we define a sequence of maps $\left\{T_{n}\right\}_{n>1}$, where each $T_{n}$ is a map $I \rightarrow$ $I, x \mapsto y=T_{n}(x)$, such that the source on the $x$-axis is divided into $p_{L}(n)$ right-open intervals of equal length, and the range on the $y$-axis is divided into $p_{L}(n-1)$ ones. The intervals of the source are then put into correspondence with the cylinders of the factors in $\operatorname{Fact}_{L}(n)$, using the lexicographic order between these factors, and the same is done for the intervals of the range with cylinders of the factors in $\operatorname{Fact}_{L}(n-1)$. Next, $T_{n}$ is defined as the piecewise affine map which sends for each $v \in \operatorname{Fact}_{L}(n)$ the interval corresponding to $\mathrm{Cyl}_{L}(v)$ to the interval corresponding to $\mathrm{Cyl}_{L}(\sigma(v))$, where $\sigma(v)$ denotes $v$ minus its first letter, by using a slope $p_{L}(n) / p_{L}(n-1)$.

First, here is a technical result about the above slopes converging to 1 . Recall that $\operatorname{sp}_{L, l}(n)$ denotes the number of left special factors in $\operatorname{Fact}_{L}(n)$, and let $\operatorname{sp}_{L, r}(n)$ denote the same for the right special factors. 
LEMMA 5.1.1. Let $L$ be a shift such that Fact $_{L}$ is prolongable. Let $\left\{n_{s}\right\}_{s \in \mathbb{N}}$ be a subsequence of $\mathbb{N} \backslash\{0,1\}$. Then $\lim _{s \rightarrow \infty}\left(p_{L}\left(n_{s}\right) / p_{L}\left(n_{s}-1\right)\right)=1$ if and only if $\lim _{s \rightarrow \infty}\left(\operatorname{sp}_{L, l}\left(n_{s}-1\right) / p_{L}\left(n_{s}-1\right)\right)=0$. The same holds for $\operatorname{sp}_{L, r}\left(n_{s}-1\right)$.

Proof. We prove the result for $\mathrm{sp}_{L, l}$ (the proof for $\mathrm{sp}_{L, r}$ is similar): $(\Rightarrow)\left(p_{L}\left(n_{s}\right) /\right.$ $\left.p_{L}\left(n_{s}-1\right)\right)-1=\left(p_{L}\left(n_{s}\right)-p_{L}\left(n_{s}-1\right)\right) / p_{L}\left(n_{s}-1\right) \geq \operatorname{sp}_{L, l}\left(n_{s}-1\right) / p_{L}\left(n_{s}-1\right) \geq$ $0,(\Leftarrow)|A| \operatorname{sp}_{L, l}\left(n_{s}-1\right) / p_{L}\left(n_{s}-1\right) \geq\left(p_{L}\left(n_{s}\right)-p_{L}\left(n_{s}-1\right)\right) / p_{L}\left(n_{s}-1\right)=\left(p_{L}\left(n_{s}\right) /\right.$ $\left.p_{L}\left(n_{s}-1\right)\right)-1 \geq 0$.

For zero entropy, using subsequences is indeed useful.

LEMMA 5.1.2. Let $L$ be a minimal aperiodic shift with zero topological entropy. Then there is a subsequence $\left\{n_{s}\right\}_{s \in \mathbb{N}}$ of $\mathbb{N} \backslash\{0,1\}$ such that $\lim _{s \rightarrow \infty}\left(p_{L}\left(n_{s}\right) / p_{L}\left(n_{s}-1\right)\right)=1$.

Proof. Since $L$ is minimal aperiodic, then for all $n>1, p_{L}(n) / p_{L}(n-1)>1$. Next, $p_{L}(n) / p_{L}(n-1)$ is not bounded away from 1. Assume on the contrary there is $c>0$ such that for all $n>1, p_{L}(n) \geq(1+c) p_{L}(n-1)$. Hence $p_{L}(n) \geq(1+c)^{n}$, and the topological entropy of $L$ would not be less than $\log (1+c)$, a contradiction.

Let $\operatorname{Fact}_{L}(u, n)=\left\{v \in \operatorname{Fact}_{L}(n) \mid v=u v^{\prime}, v^{\prime} \in A^{*}\right\}$, i.e. the set of length- $n$ factors with $u$ as prefix, and let $p_{L}(u, n)=\left|\operatorname{Fact}_{L}(u, n)\right|$. In the next result we simultaneously build measures on $L$ and approximations of the corresponding $T_{L}$.

PROPOSITION 5.1.3. Let $L$ be a minimal aperiodic shift with zero topological entropy. Let $\left\{T_{n}\right\}_{n>1}$ be the corresponding sequence of maps as defined above, and let $\mathcal{T}=\left\{T_{n_{s}}\right\}_{s \in \mathbb{N}}$, where $\left\{n_{s}\right\}_{s \in \mathbb{N}}$ is a subsequence of $\mathbb{N} \backslash\{0,1\}$ such that $\lim _{s \rightarrow \infty}\left(p_{L}\left(n_{s}\right) / p_{L}\left(n_{s}-1\right)\right)=1$. Then:

(1) there is at least one shift-invariant measure $\mu$ on $L$, induced by the $T_{n_{s}}$ 's interval lengths;

(2) there is a subsequence of $\mathcal{T}$ converging to a map $T$, equal almost everywhere to the conjugate IET $T_{L}$ obtained by Theorem 1 from $L$ measured by $\mu$.

Proof. (1) For every $s \in \mathbb{N}$ and for every $u \in \operatorname{Fact}_{L}$, we consider $p_{L}\left(u, n_{s}\right) / p_{L}\left(n_{s}\right)$, that is, the length of the interval corresponding to $\mathrm{Cyl}_{L}(u)$ on the $x$-axis of $T_{n_{s}}$. By a diagonal process on $\left\{p_{L}\left(u, n_{s}\right) / p_{L}\left(n_{s}\right)\right\}_{s \in \mathbb{N}}$, we obtain a sequence $Q=\left\{m_{i}\right\}_{i \in \mathbb{N}}$ such that, for every $u, \lim _{i \rightarrow \infty}\left(p_{L}\left(u, m_{i}\right) / p_{L}\left(m_{i}\right)\right)$ exists, and we denote it by $M_{u}$. We then define $\mu$ on the cylinders of $L$ by $\mu\left(\mathrm{Cyl}_{L}(u)\right)=M_{u}$. Note that $\mu(L)=M_{\epsilon}=1$. The finite unions of the cylinders of $A^{\mathbb{N}}$ together with the empty cylinder form a semiring of sets, thus the intersection of this semiring with the cylinders of $L$ too, and $\mu$ is a premeasure on this semiring. By Caratheodory's extension theorem, $\mu$ extends as a Borel probability measure on $L$.

Note that $\lim _{i \rightarrow \infty}\left(p_{L}\left(u, m_{i}\right) / p_{L}\left(m_{i}\right)\right)=M_{u}$ implies that $\lim _{i \rightarrow \infty}\left(p_{L}\left(u, m_{i}-1\right) /\right.$ $\left.p_{L}\left(m_{i}-1\right)\right)=M_{u} \quad$ too, that is, putting $d_{i}=\left(p_{L}\left(u, m_{i}\right) / p_{L}\left(m_{i}\right)\right)-$ $\left(p_{L}\left(u, m_{i}-1\right) / p_{L}\left(m_{i}-1\right)\right)$, we have that $\lim _{i \rightarrow \infty} d_{i}=0$. Indeed, first, since $L$ is aperiodic minimal, $p_{L}\left(m_{i}\right)>p_{L}\left(m_{i}-1\right)$ for every $i$, thus

$$
d_{i}<\frac{p_{L}\left(u, m_{i}\right)}{p_{L}\left(m_{i}-1\right)}-\frac{p_{L}\left(u, m_{i}-1\right)}{p_{L}\left(m_{i}-1\right)} \leq|A| \frac{\operatorname{sp}_{L, l}\left(m_{i}-1\right)}{p_{L}\left(m_{i}-1\right)},
$$


which, by Lemma 5.1.1, converges to 0 . Next, since $p_{L}\left(u, m_{i}\right) \geq p_{L}\left(u, m_{i}-1\right)$,

$$
\begin{aligned}
d_{i} & \geq \frac{p_{L}\left(u, m_{i}-1\right)}{p_{L}\left(m_{i}\right)}-\frac{p_{L}\left(u, m_{i}-1\right)}{p_{L}\left(m_{i}-1\right)}=p_{L}\left(u, m_{i}-1\right) \frac{p_{L}\left(m_{i}-1\right)-p_{L}\left(m_{i}\right)}{p_{L}\left(m_{i}\right) p_{L}\left(m_{i}-1\right)} \\
& \geq-p_{L}\left(u, m_{i}-1\right)|A| \frac{\mathrm{sp}_{L, l}\left(m_{i}-1\right)}{p_{L}\left(m_{i}\right) p_{L}\left(m_{i}-1\right)}=-|A| \frac{p_{L}\left(u, m_{i}-1\right)}{p_{L}\left(m_{i}\right)} \frac{\mathrm{sp}_{L, l}\left(m_{i}-1\right)}{p_{L}\left(m_{i}-1\right)},
\end{aligned}
$$

which also converges to 0 , again by Lemma 5.1.1.

We now check the preservation of $\mu$ by $\sigma$. Assume that the letter prolongations in Fact $_{L}$ of $u$ to the left are $a_{1}, \ldots, a_{h} \in A$, where $h>0$ since Fact $_{L}$ is prolongable, $L$ being minimal. Thus $\sigma^{-1}\left(\mathrm{Cyl}_{L}(u)\right)=\bigcup_{j=1}^{h} \operatorname{Cyl}_{L}\left(a_{j} u\right)$. For all $m_{i} \in Q$, we also have $\sum_{j=1}^{h} p_{L}\left(a_{j} u, m_{i}\right) \geq p_{L}\left(u, m_{i}-1\right)$, and the difference between these two terms depends on the number of left special factors in $\operatorname{Fact}_{L}\left(u, m_{i}-1\right)$ and on the number of their prolongations:

$$
0 \leq \sum_{j=1}^{h} p_{L}\left(a_{j} u, m_{i}\right)-p_{L}\left(u, m_{i}-1\right) \leq|A| \operatorname{sp}_{L, l}\left(m_{i}-1\right) .
$$

Similarly, for each $a_{j}, p_{L}\left(a_{j} u, m_{i}\right) \geq p_{L}\left(a_{j} u, m_{i}-1\right)$, and the difference between these terms depends on the number of right special factors in $\operatorname{Fact}_{L}\left(a_{j} u, m_{i}-1\right)$ :

$$
0 \leq \sum_{j=1}^{h} p_{L}\left(a_{j} u, m_{i}\right)-\sum_{j=1}^{h} p_{L}\left(a_{j} u, m_{i}-1\right) \leq|A| \operatorname{sp}_{L, r}\left(m_{i}-1\right) .
$$

Reversing (11) and adding it to (10), for all $m_{i} \in Q$, we get

$$
-|A| \operatorname{sp}_{L, r}\left(m_{i}-1\right) \leq \sum_{j=1}^{h} p_{L}\left(a_{j} u, m_{i}-1\right)-p_{L}\left(u, m_{i}-1\right) \leq|A| \operatorname{sp}_{L, l}\left(m_{i}-1\right) .
$$

We then divide the above terms by $p_{L}\left(m_{i}-1\right)$, let $i$ go to infinity, and using Lemma 5.1.1 again, we obtain $\mu\left(\mathrm{Cyl}_{L}(u)\right)=\sum_{j=1}^{h} \mu\left(\mathrm{Cyl}_{L}\left(a_{j} u\right)\right)$. Hence $\mu$ is an invariant measure for $L$. Moreover, since $L$ is aperiodic minimal, $\mu$ is non-atomic.

(2) On the $x$-axis for each $u \in$ Fact $_{L}$, the length of the interval corresponding to $\operatorname{Cyl}_{L}(u)$ converges to $M_{u}$ as $\lim _{i \rightarrow \infty}\left(p_{L}\left(u, m_{i}\right) / p_{L}\left(m_{i}\right)\right)$, and the same is true on the $y$-axis for $\sigma(u)$ with $M_{\sigma(u)}$ as $\lim _{i \rightarrow \infty}\left(p_{L}\left(\sigma(u), m_{i}-1\right) / p_{L}\left(m_{i}-1\right)\right)$. Now, since $L$ has zero topological entropy, and $\mu$ has all the requested properties, Theorem 1 applies to $L$ measured by $\mu$, so as to obtain $T_{L}$, with its associated partitions $\mathrm{PART}_{L}$ and $\mathrm{PART}_{L, I}$. The main components of $\mathrm{PART}_{L}$ are the cylinders $\mathrm{Cyl}_{L}\left(v^{(k)}\right)$ (see Lemma 3.2.5), and following the construction of $T_{m_{i}}$, on the $x$-axis for each $k$, the associated intervals $I_{m_{i}}^{(k)}$ are the union of the intervals corresponding to $\operatorname{Cyl}_{L}\left(v^{(k)} v\right), v^{(k)} v \in \operatorname{Fact}_{L}\left(m_{i}\right)$, then converging to an interval $I^{(k)}$ of length $M_{v^{(k)}}$. The same holds for the intervals $J_{m_{i}}^{(k)}$ in the $y$-axis with $\mathrm{Cyl}_{L}\left(\sigma\left(v^{(k)} v\right)\right)$, respectively converging to intervals $J^{(k)}$ of length $M_{\sigma\left(v^{(k)}\right)}$. Note then that for every $i, T_{m_{i}}$ is continuous, hence is an affine map on each $I_{m_{i}}^{(k)}$. Indeed, $\sigma$ is increasing on $\mathrm{Cyl}_{L}\left(\sigma\left(v^{(k)}\right)\right)$, thus $T_{m_{i}}$ on $I_{m_{i}}^{(k)}$ too. But if $T_{m_{i}}$ had a discontinuity, it would mean that $\sigma^{-1}$ is not well-defined in this cylinder, contradicting the definition of $\mathrm{PART}_{L}$. 
Now, the left endpoint of $I^{(k)}$ is $o_{k}=\sum_{v<v^{(k)}, v \in \operatorname{Fact}_{L}\left(\left|v^{(k)}\right|\right)} M_{v}$, and the lower endpoint of $J^{(k)}$ is $o_{k}^{\prime}=\sum_{v<\sigma\left(v^{(k)}\right), v \in \operatorname{Fact}_{L}\left(\left|v^{(k)}\right|-1\right)} M_{v}$. By convergence, for every $k$ and $\varepsilon>0$, there is $j>0$ such that for all $i \geq j$, the endpoints of $I_{m_{i}}^{(k)}$ are at distances $<\varepsilon$ respectively from $o_{k}$ and $o_{k}+M_{v^{(k)}}$. Thus let $T_{i, k, \varepsilon}$ be $T_{m_{i}}$ restricted to $I_{\epsilon}^{(k)}=\left[o_{k}+\varepsilon, o_{k}+M_{v^{(k)}}-\varepsilon\right]$ for $i \geq j$, on which it is affine with slope $p_{L}\left(m_{i}\right) / p_{L}\left(m_{i}-1\right)$. These slopes converge to 1 , and the endpoints of $J_{m_{i}}^{(k)}$ converge respectively to $o_{k}^{\prime}$ and $o_{k}^{\prime}+M_{\sigma\left(\mathrm{Cyl}_{L}\left(v^{(k)}\right)\right)}$, hence $T_{i, k, \varepsilon}$ converges on $I_{\epsilon}^{(k)}$. Since $\varepsilon$ is arbitrary, $\left\{T_{m_{i}}\right\}_{i \in \mathbb{N}}$ converges on all the $I^{(k)^{o}}$, i.e. the $I^{(k)}$ interiors. We put $T$ as the limit map, so that for each $x \in I^{(k)^{o}}, T(x)=o_{k}^{\prime}+\left(x-o_{k}\right)$.

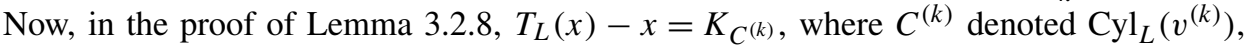
and where $K_{C^{(k)}}=\mu\left(\left[w_{L, \min }, \sigma\left(w_{C^{(k)}, \min }\right)\right]\right)-\mu\left(\left[w_{L, \min }, w_{C^{(k)}, \min }\right]\right)$. By the definition of $T$, the first term of this difference is $o_{k}^{\prime}$ and the second is $o_{k}$, that is, $T_{L}(x)-x=T(x)-$ $x$ on $I^{(k)^{o}}$, thus by comparison with Lemma 3.2.8, $I^{(k)^{o}}=I_{L}^{(k)^{o}}$. Since by Lemma 3.2.10, $\mu\left(\mathrm{SP}_{L}\right)=0$, the union of the $I^{(k)^{o}}$ has measure 1 , whence $T=T_{L}$ almost everywhere.

Note that the map $T$ above can be extended to all of $I$ by right-continuity, so that $T$ can be made equal to $T_{L}$.

5.2. Explicit graph examples. A dynamical system $(X, f)$ is said to be uniquely ergodic if there exists only one $f$-invariant Borel probability measure on $X$.

PROPOSITION 5.2.1. Let $L$ be an aperiodic minimal shift with linear complexity which is uniquely ergodic. Then $\mathcal{T}$ in Proposition 5.1.3, defined as $\left\{T_{n}\right\}_{n>1}$, converges almost everywhere to $T_{L}$.

Proof. Since $L$ has linear complexity, its topological entropy is zero. For the same reason, there exists $r>0$ such that for all $n>1, p_{L}(n)-p_{L}(n-1) \leq r$ [Cas96], hence $\lim _{n \rightarrow \infty}\left(p_{L}(n) / p_{L}(n-1)\right)=1$, and Proposition 5.1.3 can be applied to $L$. Now, in the proof of this proposition, unique ergodicity means there can be only one accumulation point for each $\left\{p_{L}(u, n) / p_{L}(n)\right\}_{n>1}, u \in$ Fact $_{L}$. Thus these sequences converge without any subsequence extraction, as then does $\mathcal{T}$.

In order to illustrate the above proposition, the simplest non-trivial examples are given by the primitive substitutive case (see p. 1945).

COROLLARY 5.2.2. Let $L$ be a shift associated with an aperiodic primitive substitution. Then Proposition 5.2.1 applies to L.

Proof. Aperiodic primitive substitutions have associated shifts which are aperiodic minimal with linear complexity [Pan84], and uniquely ergodic [Qué10].

Note that in this primitive substitutive case, the limit measure of each cylinder can also be computed from the substitution itself [Qué10, § 5].

Example 5.2.3. Reconsider the Thue-Morse substitution $\theta_{t m}$ (see Examples 3.2.6, 3.2.11, and 3.2.12). Since $\theta_{t m}$ is aperiodic primitive, Corollary 5.2.2 applies to its associated shift $L_{t m}$. In the following picture we show the graph of $T_{100}$ from the converging sequence $\mathcal{T}=\left\{T_{n}\right\}_{n>1}$ approximating the IET $T_{L_{t m}}$ with $B_{L_{t m}}$ as set of separation points. 
The intervals converging to their respective $I_{L_{t m}}^{(k)}$ of $\mathrm{PART}_{L_{t m}, I}$ are indicated for the first $k$, by the associated $v^{(k)}$ determining the corresponding cylinders of $\operatorname{PART}_{L_{t m}}$ (see Lemma 3.2.5 and Example 3.2.6). As expected from the Examples 3.2.11 and 3.2.12, two accumulations of discontinuity points can be observed, where non-injectivity holds. Note first that, in this Thue-Morse case, the limit measure of each involved cylinder can be directly obtained from [Dek92]. Second, this example suggests existing connections with constructions of infinite permutations obtained from shifts associated with aperiodic primitive substitutions [Mak09, AFP15].

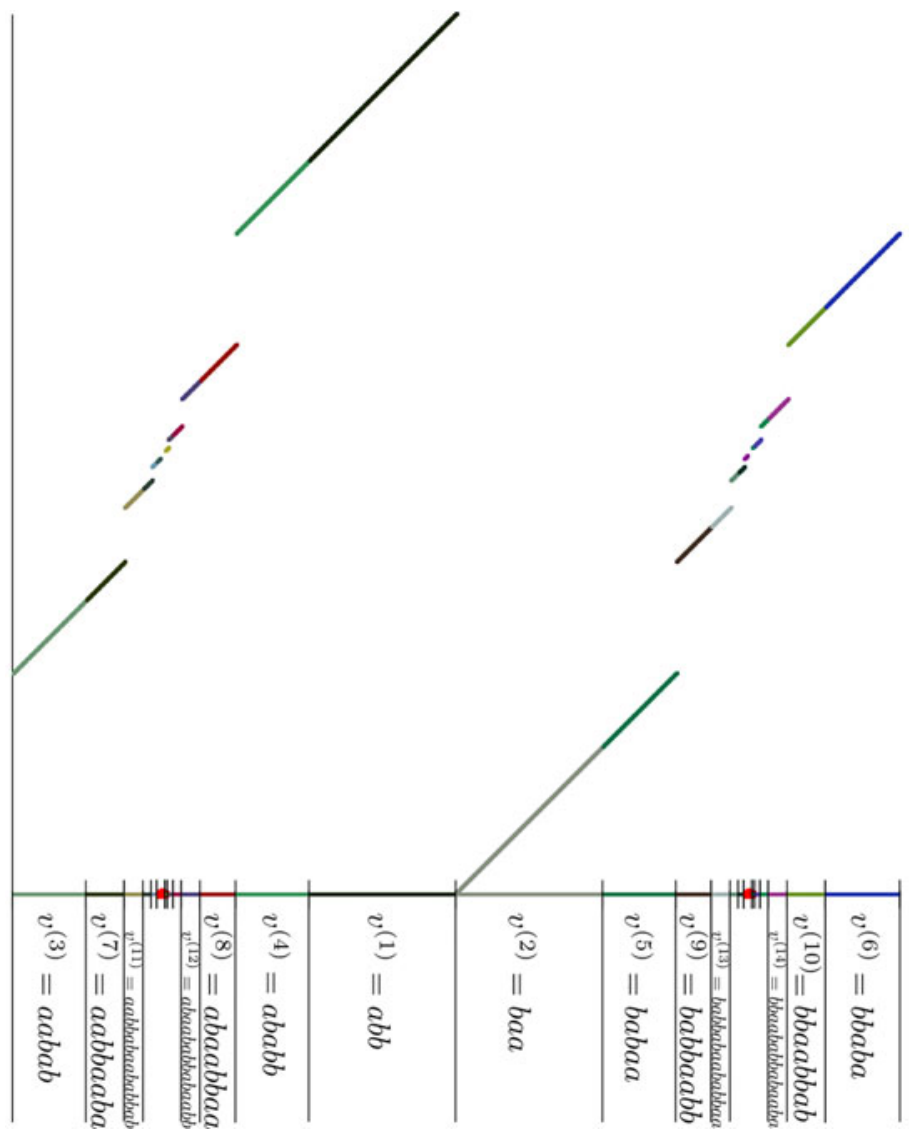

Example 5.2.4. Here are other examples of approximated infinite IETs from linear complexity shifts associated with aperiodic primitive substitutions. The pictures below are the $T_{100}$ graphs from the convergent sequences $\mathcal{T}=\left\{T_{n}\right\}_{n>1}$ given by Corollary 5.2.2 for: (i) the Tribonacci substitution, i.e. $\theta(a)=a b, \theta(b)=a c, \theta(c)=a$; (ii) the Tetranacci substitution, i.e. $\theta(a)=a b, \theta(b)=a c, \theta(c)=a d, \theta(d)=a$; (iii) the RudinShapiro substitution, i.e. $\theta(a)=a b, \theta(b)=a c, \theta(c)=d b, \theta(d)=d c$; (iv) the Fibonacci substitution, i.e. $\theta(a)=a b, \theta(b)=a$. In this last example, $L_{\theta}$ is known to be the shift given by the natural coding of the minimal finite IET $T$ over two intervals, where $B=\mathcal{D}=\{0,1 / \rho\}$, and $\rho$ is the golden ratio [Fog02]. Note then that in accordance with 
Corollary 3.3.4, the drawn graph is an approximation of the graph of $T$ and of the graph of $T_{L_{\theta}}$ too, while the construction of $T_{L_{\theta}}$ describes it with an infinite set $B_{L_{\theta}}$ of separation points.

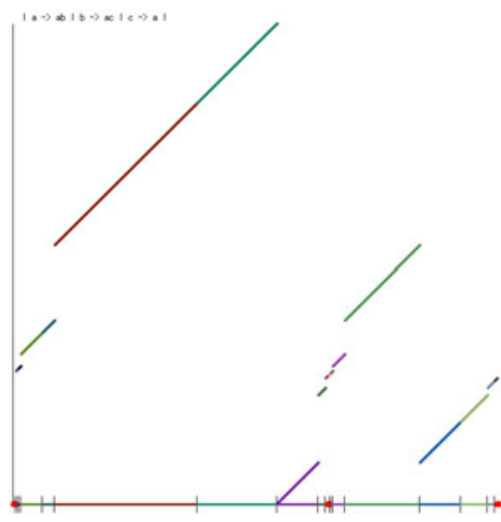

(i) Tribonacci

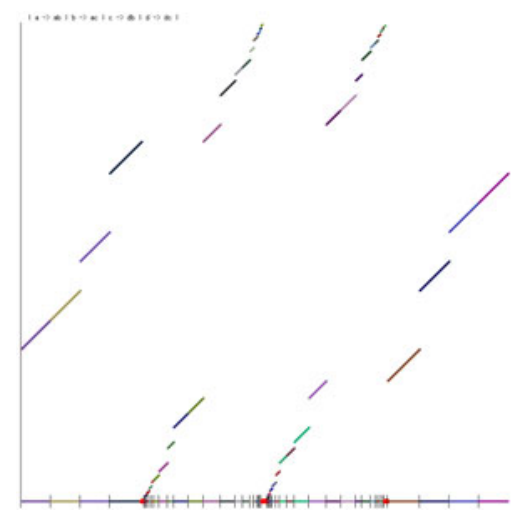

(iii) Rudin-Shapiro

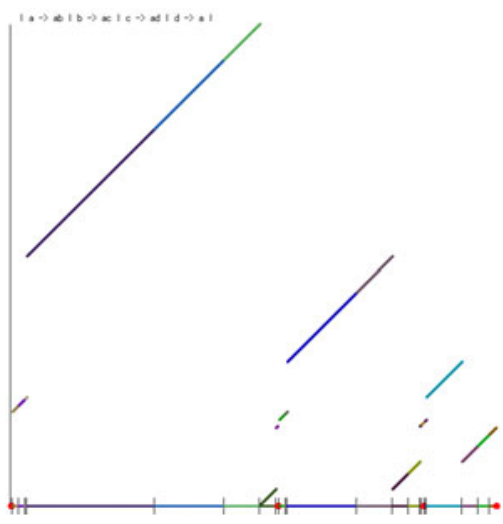

(ii) Tetranacci

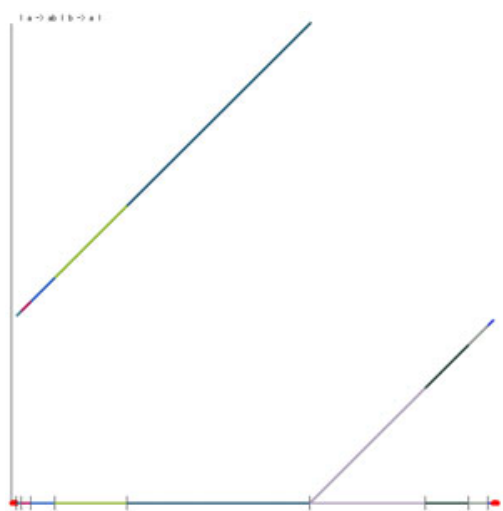

(iv) Fibonacci

\section{REFERENCES}

[AFP15] S. V. Avgustinovich, A. E. Frid and S. Puzynina. Canonical representatives of morphic permutations. WORDS'15 (Lecture Notes in Computer Science, 9304). Springer, Berlin, 2015, pp. 59-72.

[AOW85] P. Arnoux, D. Ornstein and B. Weiss. Cutting and stacking, interval exchanges and geometric models. Israel J. Math. 50(1-2) (1985), 160-168.

[Cas96] J. Cassaigne. Special factors of sequences with linear subword complexity. Developments in Language Theory, II (Magdeburg, 1995). World Sci. Publ., River Edge, NJ, 1996, pp. 25-34.

[CFS82] I. P. Cornfeld, S. V. Fomin and Y. G. Sinaĭ. Ergodic Theory Grundlehren der Mathematischen Wissenschaften (Fundamental Principles of Mathematical Sciences, 245). Springer, New York, 1982.

[CN10] J. Cassaigne and F. Nicolas. Factor complexity. Combinatorics, Automata and Number Theory (Encyclopedia Mathematics and its Applications, 135). Eds. V. Berthé and M. Rigo. Cambridge Unversity Press, Cambridge, 2010, pp. 163-247.

[Dek92] F. M. Dekking. On the Thue-Morse measure. Acta Univ. Carolin. Math. Phys. 33(2) (1992), 35-40. 
[ER83] A. Ehrenfeucht and G. Rozenberg. Repetitions of subwords in D0L languages. Inform. Control 59 (1983), 13-35.

[Fog02] N. Pytheas Fogg. Substitutions in Dynamics, Arithmetics and Combinatorics (Lecture Notes in Mathematics, 1794). Eds. V. Berthé, S. Ferenczi, C. Mauduit and A. Siegel. Springer, Berlin, 2002.

[HK02] B. Hasselblatt and A. Katok. Principal structures. Handbook of Dynamical Systems. Vol. 1A. North-Holland, Amsterdam, 2002, pp. 1-203.

[Hoo15] W. P. Hooper. The invariant measures of some infinite interval exchange maps. Geom. Topol. 19 (2015), 1895-2038.

[Kea75] M. Keane. Interval exchange transformations. Math. Z. 141 (1975), 25-31.

[Kit98] B. P. Kitchens. Symbolic Dynamics. Springer, Berlin, 1998.

[KST01] S. Kolyada, L. Snoha and S. Trofimchuk. Noninvertible minimal maps. Fund. Math. 168(2) (2001), 141-163.

[LM95] D. Lind and B. Marcus. Symbolic Dynamics and Coding. Cambridge Unversity Press, Cambridge, 1995.

[Mak09] M. A. Makarov. On an infinite permutation similar to the Thue-Morse word. Discrete Math. 309(23-24) (2009), 6641-6643.

[Mañ87] R. Mañé. Ergodic Theory and Differentiable Dynamics. Springer, Berlin, 1987.

[MH38] M. Morse and G. A. Hedlund. Symbolic dynamics I. Amer. J. Math. 60 (1938), 815-866.

[Pan84] J.-J. Pansiot. Complexité des facteurs des mots infinis engendrés par morphismes itérés. ICALP'84 (Lecture Notes in Computer Science, 172). Springer, Berlin, 1984, pp. 380-389.

[Par66] W. Parry. Symbolic dynamics and transformations of the unit interval. Trans. Amer. Math. Soc. 122 (1966), 368-378.

[Qué10] M. Quéffelec. Substitution Dynamical Systems-Spectral Analysis (Lecture Notes in Mathematics, 1294). 2nd edn. Springer, Berlin, 2010.

[Thu12] A. Thue. Über die gegenseitige Lage gleicher Teile gewisser Zeichenreihen [On the relative position of equal parts in certain sequences of symbols]. Kra. Vidensk. Selsk. Skrifter. I. Mat.-Nat. Kl. 10 (1912), 1-67. Reprinted in Selected Mathematical Papers of Axel Thue. Ed. T. Nagell et al. Universitetsforlaget, Oslo, 1977, pp. 413-478. 